\title{
Iterative Nonlinear Self-Interference Cancellation for In-Band Full-Duplex Wireless Communications Under Mixer Imbalance and Amplifier Nonlinearity
}

\author{
Kazuki Komatsu, Student Member, IEEE, Yuichi Miyaji, Member, IEEE, and Hideyuki Uehara, Member, IEEE
}

\begin{abstract}
This paper presents an iterative estimation and cancellation technique for nonlinear in-band full-duplex transceivers with IQ imbalances and amplifier nonlinearities. The estimation process of the proposed scheme consists of three stages, namely, the channel response estimation, IQ imbalance estimation, and power amplifier and low-noise amplifier (LNA) nonlinearities estimation. For the estimation of the parameters and improvement of the accuracy, distortions are compensated by cancellation or inversion with the latest estimated parameters. On the one hand, the channel response is estimated on the time domain; on the other hand, the IQ imbalance and nonlinearities are estimated on the frequency domain for a more straightforward estimation and superior accuracy. In the cancellation process of the proposed scheme, the received signal is compensated with the estimated parameters of the LNA and receiver IQ imbalance before cancellation because the desired signal is received with a high-power self-interference and is distorted by the radiofrequency receiver impairments. Simulation results show that the proposed technique can achieve higher cancellation performance compared with the Hammerstein canceller when the LNA is saturated by the self-interference. Additionally, the performance of the proposed canceller converges much faster than that of the Hammerstein canceller.
\end{abstract}

Index Terms-Full-duplex radio, self-interference, digital cancellation, IQ imbalance, amplifier nonlinearity.

\section{INTRODUCTION}

I N-band full-duplex communications, which transmit and receive information at the same time and same frequency band, have the capability to increase the channel capacity of conventional wireless communication systems [1]. Fullduplex communication can replace the conventional halfduplex communication system, and its application to various communication systems, such as wireless local area networks (WLAN) [2], [3], device-to-device relay communications [4], [5], cellular networks [6], [7], and self-backhauling systems [8], has been studied. Self-interference is the most challenging obstacle to realize full-duplex communications, and it is important to reduce the self-interference to the thermal noise level. Generally, the self-interference signal is gradually eliminated by introducing antenna isolation and self-interference

This work was supported by the Hoso Bunka Foundation and the Japan Society for the Promotion of Science (JSPS) KAKENHI Grant Numbers JP18K04138, JP19K14979, and JP19J12727.

In this work, we used the cluster computer system of the Information Media Center (IMC), Toyohashi University of Technology, and the supercomputer of the Academic Center for Computing and Media Studies (ACCMS), Kyoto University.

The authors are with the Department of Electrical and Electronic Information Engineering, Toyohashi University of Technology, Japan (e-mail: komatsu.kazuki.op@tut.jp; miyaji@ee.tut.ac.jp; uehara@tut.jp). cancellation in the analog radio frequency (RF) domain and the digital baseband domain [9], [10]. For example, if the transmission power is $20 \mathrm{dBm}$ and the thermal noise level is $-90 \mathrm{dBm}$, the total performance of all self-interference cancellation stages needs to reach $110 \mathrm{~dB}$. Furthermore, it is difficult to achieve high self-interference cancellation due to the constraints of systems and the nonlinearity of analog circuits.

\section{A. Review of Previous Research: Analog Approach}

Whenever the low-noise amplifier (LNA) and analog-todigital converter (ADC) of the receiver are saturated due to strong self-interference, eliminating self-interference in digital baseband becomes difficult. Thus, sufficient self-interference cancellation in the analog stage is necessary to eliminate the self-interference signal. For example, if the transmission power is $20 \mathrm{dBm}$ and the saturated input level of the LNA is $-10 \mathrm{dBm}$, the analog cancellation needs to be at least $40 \mathrm{~dB}$ to guarantee a $10 \mathrm{~dB}$ peek-to-average-power ratio (PAPR). In the literature [11]-[21], two major ways to reduce the selfinterference signal in the analog domain have been proposed.

The first step for suppressing self-interference is to increase the transceiver isolation between the transmitter and receiver chains. In full-duplex systems, two methods to accomplish this exist: sharing an antenna for transmission and reception [12], and separating antennas [13]. In the antenna-sharing method, isolation between transmitters and receivers is only approximately $15 \mathrm{~dB}$ [12] because it depends on the circulator's isolation. In antenna-separation systems, high isolation can be achieved by orthogonalization of the polarization and direction, or by increasing the distance between the antennas [11]. However, in small terminals, such as smartphones and IoT devices, the amount of isolation is reduced owing to size restrictions. The equivalent baseband models for these two configurations are the same, although they have different characteristics. Accordingly, we do not impose any restrictions on the antenna configuration in this study.

The second step for suppressing self-interference is analog RF-domain cancellation, which is performed to prevent saturation in the receiver LNA and ADC. The most researched analog cancellation scheme [1], [12]-[16] is a multi-tapped delay-line RF canceller that consists of digital controllers and passive elements, such as splitters, variable attenuators, variable phase shifters, and delay lines. This delay-line canceller can remove both the linear self-interference signal and nonlinear components of the power amplifier (PA) output signal 
because the canceller uses signals obtained by linear conversion of the PA output signal. We can configure this canceller flexibly because the number of delay lines can be increased or decreased depending on the system specifications, cost requirements, and size restrictions. Furthermore, an analogdomain cancellation scheme with an auxiliary transmitter has been proposed [17]-[21]. This canceller can take into account multipath fading and transmitter IQ imbalance by including strong signal processing. However, this canceller may not be suitable for cheap terminals because it requires an additional transmitter. In this paper, we assume that terminals use a simple delay-line canceller to mitigate the direct-path selfinterference signal only because they are constrained by costs and size requirements.

\section{B. Review of Previous Research: Digital Approach}

Digital self-interference cancellation is the final step of a series of cancellation processes. With powerful digital signal processing techniques, digital cancellers with various features have been developed, as shown in TABLE I. In this field, it is common to deal with RF impairments [12], [16], [22][29], [34], [37], [38], phase noise [35], [36], and application to multi-input and multi-output (MIMO) systems [24], [25], [40], [41], and application of blind signal processing [42], [43]. In addition to the research focused on digital cancellers composed only of digital signal processing, there are also studies integrating auxiliary receivers [31]-[33].

The simplest digital canceller is the time-domain linear canceller, which has one finite impulse response (FIR) filter. The linear canceller can regenerate the linear component of the self-interference, but its performance is considerably degraded by the nonlinearity of the transceiver. In general, inexpensive terminals suffer from nonlinearity problems more than expensive terminals. The most problematic RF impairments are the IQ mixer imbalance and PA nonlinearity [31]. To deal with these impairments, Hammerstein cancellers [12], [16], [23][30] have been developed. This type of canceller estimates channel responses for all nonlinear basis functions by applying a least-squares estimation. A digital canceller with an auxiliary receiver [31]-[33], which is used to receive the output signal of the PA directly, can perfectly regenerate the nonlinear self-interference signal caused by the transmitter nonlinearities using lightweight digital signal processing. However, the nonlinearity due to the receiver LNA cannot be expressed by the Hammerstein model, and the Hammerstein canceller and the auxiliary receiver cannot cope with the nonlinear distortion of the LNA. In literature [34], a canceller that addresses the nonlinearity of both the PA and LNA has been developed. This canceller estimates the radio channel and the nonlinear characteristics alternately. However, IQ imbalance is not considered in this scheme. In other works [44]-[47], mitigation techniques of receiver nonlinearity were proposed for wideband receivers. These techniques do not employ selfinterference cancellers and cannot deal with the nonlinear distortion of the transmitter. To use these techniques for selfinterference cancellation, it is necessary to complement them with other cancellers that can mitigate the nonlinear distortion of the transmitter. The Volterra series-based cancellers [37][39] have the potential to mitigate the nonlinearities of the PA and LNA and the IQ imbalance. However, they need significantly higher computational power to estimate and regenerate the nonlinear self-interference signal than other practical cancellers. For example, the Hammerstein canceller and the canceller described in [37] need to estimate $12 M=576$ and $7 M^{3}+3 M^{2}+2 M+1 \approx 7.81 \times 10^{5}$ parameters for fifthorder nonlinearity cancellation, respectively, when the number of taps of the channel impulse response is $M=48$. To the best of our knowledge, a digital self-interference canceller that addresses the nonlinearities of the PA and LNA, and the imbalance of IQ mixers with reasonable computational cost has not been developed yet. The development of such a canceller would enable the construction of inexpensive fullduplex terminals.

In systems where the transmitter and receiver do not share a local oscillator, phase noise will be a major obstacle in addition to RF impairments. Estimating the time-varying phase noise is generally difficult and challenging. In [35], a linear digital canceller with phase noise estimation have been proposed. This canceller estimates the phase noise with the minimum mean square error (MMSE) algorithm under the presence of the desired signal. Similarly, in [36], the phase noise was estimated with the maximum likelihood algorithm, and then the nonlinear self-interference was reconstructed and removed.

To achieve higher channel capacity on in-band full-duplex communications, cancellers should have a lower number of training symbols. For example, self-interference cancellers with blind signal processing have been proposed on orthogonal frequency-division multiplexing (OFDM) systems [42], [43]. However, these techniques require symbol synchronization between the self-interference and the desired signal because they are applied in the frequency domain [43]. This problem is inherent in frequency-domain cancellers and limits the location of two full-duplex terminals that communicate with each other. Hammerstein cancellers with frequency-domain cancellation have the same problem, and time-domain cancellation is required to avoid this issue [28].

Currently, the most common communication systems are MIMO systems, and the application of digital cancellers to these systems is an important subject. Some studies [24], [25] have been conducted to upgrade single-input and singleoutput (SISO) Hammerstein cancellers to MIMO systems. Unlike SISO systems, MIMO systems can use extra spatial dimensions, and many received signals for self-interference cancellation. A precoding scheme [40], [48]-[50] has been developed to optimize the receiver weight matrix and transmitter precoder matrix for reducing self-interference. In [41], it was mentioned that, on a massive MIMO system, the selfinterference can be reduced by a zero-forcing receiver with a large number of transmitting and receiving antennas. Some research has been done on full-duplex relay systems to mitigate self-interference and maximize spectral efficiency [51], [52]. In [51], a transmission power optimization technique was proposed for full-duplex multi-antenna relay systems. In [52], the conditions under which half-duplex or full-duplex mode have higher spectral efficiency and an opportunistic 
TABLE I

Digital CANCELLERS AND RF IMPAIRMENTS IN THE LiTERATURE

\begin{tabular}{c|c|c|c|c}
\hline \hline & \multicolumn{4}{|c}{ Addressed RF Impairments } \\
\hline & IQ Imbalance & PA Nonlinearity & LNA Nonlinearity & Phase Noise \\
\hline Linear [13] & & & & \\
\hline Widely Linear [22] & $\checkmark$ & & & \\
\hline Hammerstein [12], [16], [23]-[30] & $\checkmark$ & $\checkmark$ & & \\
\hline Auxiliary Receiver [31]-[33] & $\checkmark$ & $\checkmark$ & & \\
\hline [34] & & $\checkmark$ & $\checkmark$ & $\checkmark$ \\
\hline [35] & & & & \\
\hline [36] & $\checkmark$ & $\checkmark$ & $\checkmark$ & \\
\hline Volterra Series [37]-[39] & $\checkmark$ & $\checkmark$ & $\checkmark$ & \\
\hline Our Proposed & $\checkmark$ & $\checkmark$ & &
\end{tabular}

mode selection scheme were studied. Precoding techniques of MIMO systems and power optimization techniques of relays are used in conjunction with digital self-interference cancellers. Thus, developing a digital canceller with better performance is also important in these systems.

\section{Our Contributions}

In small and inexpensive terminals, the performance of RF cancellers will be constrained in terms of size and cost. Therefore, the RF canceller cannot sufficiently remove the self-interference, and the nonlinearity of the LNA will be a problem to achieve in-band full-duplex communications. However, a practical digital self-interference canceller that takes into account the nonlinearities of both the LNA and PA and the mixer imbalance has not been developed yet. The contributions of this study are as follows:

- To develop a novel digital canceller, we define operators that characterize the nonlinearity of the self-interference signal. Even if the self-interference signal contains complex nonlinearities, the signal model with these operators tells us what operations are needed to estimate the parameters and eliminate self-interference.

- By referring to the nonlinear signal model with operators, we introduce a novel estimation and cancellation scheme of the self-interference signal that can deal with the nonlinearity of the LNA. The estimation process of the proposed scheme is divided into three stages: IQ imbalance estimation, channel impulse response estimation, and PA and LNA nonlinearities estimation. These stages are executed iteratively in order, and the estimated parameters converge to better values. The proposed scheme can achieve high cancellation performance with much fewer learning symbols compared to Hammerstein cancellers because it estimates the simplest and smallest parameters that are sufficient to represent the nonlinear self-interference model.

- To achieve the best self-interference cancellation, the canceller should apply the inverse distortion of the receiver to the received signal. Conventional methods cannot generate the inverse characteristic of the receiver nonlinearity because they do not estimate the nonlinear characteristics of the transceiver and the characteristics of the channel separately. In contrast, the proposed method can generate the inverse characteristic of each distortion because the nonlinear distortion characteristics and channel impulse response are estimated separately. Thus, the proposed canceller applies a post-distortion to the received signal to compensate for the receiver RF impairments before self-interference cancellation. In addition, to avoid the synchronization problem of frequency-domain cancellers, these series of cancellations are processed in the time domain.

The rest of this paper is organized as follows. In Section II, a detailed model of the self-interference, which includes the nonlinearities of IQ mixers and amplifiers of the transceiver, and the mathematical operators that represent the inputoutput characteristic of each component are provided. The proposed estimation and cancellation scheme are presented in Section III. In Section IV, the performance of the proposed scheme under different scenarios is analyzed with equivalent baseband signal simulations. Finally, this paper is concluded in Section V.

\section{Self-Interference Signal Model}

The signal model used in this study is the same as that reported in [22], [28]. In this section, we describe the discretetime and discrete-frequency baseband equivalent signal models corresponding to the input-output characteristics of each nonlinear RF components. The properties of each component are provided as mathematical operators that only consist of sufficient parameters to represent their nonlinear properties. In Section III, these operators are used to explain the proposed method. We assume that there is no signal other than the selfinterference in the training period of the canceller. Hence, in this section, we focus on the self-interference signal only.

\section{A. Transmit Signal}

The transmit OFDM signal with $N_{\mathrm{sc}}$ subcarriers is described as

$$
x[n]=\sum_{\substack{k=-N_{\mathrm{sc}} / 2 \\ k \neq 0}}^{N_{\mathrm{sc}} / 2} X_{i}[k] e^{j 2 \pi k \Delta f n T_{s}},
$$

where $X_{i}[k]$ is the $k$ th subcarrier at the $i$ th OFDM symbol, and $\Delta f$ and $T_{s}$ are the frequency interval of each subcarrier and the sampling interval of the system, respectively. To estimate and remove the nonlinear self-interference signal, the bandwidth of the baseband signal processing, which is 


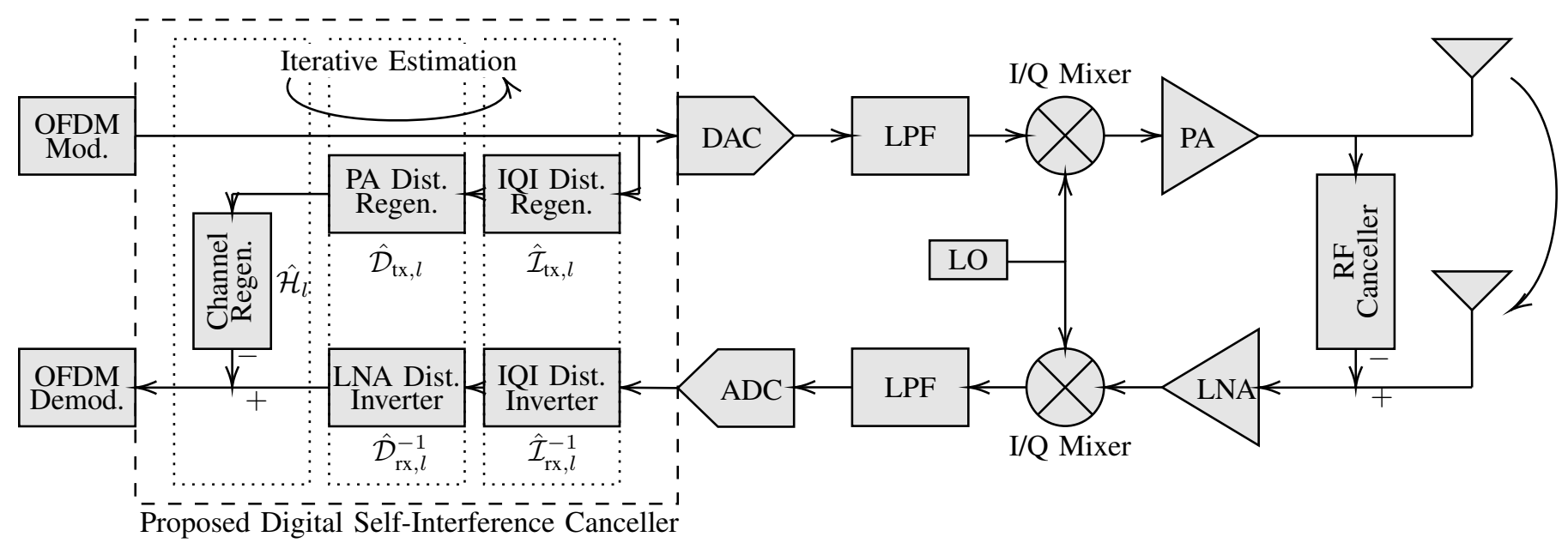

Fig. 1. In-band full-duplex transceiver model with the proposed digital self-interference canceller

expressed as $1 / T_{s}$, needs to be larger than $P N_{\mathrm{sc}} \Delta f$, where $P$ is the maximum nonlinear order of the canceller.

\section{B. Imbalance of $I Q$ Mixers}

The transmit signal $x[n]$ is upconverted from the baseband to the RF band on the IQ mixer of the transmitter. The IQ imbalance is one of the most significant distortions in directconversion transceivers, and it has been discussed extensively in the literature [20], [22], [53]. The frequency-independent imbalance model is described as

$$
x_{\mathrm{IQ}}[n]=c_{\mathrm{tx}, 1} x[n]+c_{\mathrm{tx}, 2} x^{*}[n]=c_{\mathrm{tx}, 1} \mathcal{I}_{\mathrm{tx}} x[n],
$$

where $c_{\mathrm{tx}, 1}$ and $c_{\mathrm{tx}, 2}$ are the coefficients of the input signal and conjugated signal, respectively. To simplify the explanation of the proposed scheme in Section III, we define the operator $\mathcal{I}_{\text {tx }}$ as follows:

$$
\begin{aligned}
& \mathcal{I}_{\mathrm{tx}} s[n]=s[n]+\frac{c_{\mathrm{tx}, 2}}{c_{\mathrm{tx}, 1}} s^{*}[n]=s[n]+b_{\mathrm{tx}} s^{*}[n], \\
& \mathcal{I}_{\mathrm{tx}} S_{i}[k]=S_{i}[k]+\frac{c_{\mathrm{tx}, 2}}{c_{\mathrm{tx}, 1}} S_{i}^{*}[-k]=S_{i}[k]+b_{\mathrm{tx}} S_{i}^{*}[-k],
\end{aligned}
$$

where $s[n]$ and $S_{i}[k]$ are baseband signals, and $b_{\mathrm{tx}}=$ $c_{\mathrm{tx}, 2} / c_{\mathrm{tx}, 1}$. In (3), the linear amplification factor is not included in the definition of the operator $\mathcal{I}_{\text {tx }}$ because we want it to express the characteristics of IQ imbalance to a minimum form. The image rejection rate, which is an indicator of the IQ imbalance, is defined as

$$
\operatorname{IRR}_{\mathrm{tx}}=\frac{\left|c_{\mathrm{tx}, 1}\right|^{2}}{\left|c_{\mathrm{tx}, 2}\right|^{2}}=\left|b_{\mathrm{tx}}\right|^{-2} .
$$

For the receiver IQ mixer, we also define $c_{\mathrm{rx}, 1}, c_{\mathrm{rx}, 2}$ and $\mathrm{IRR}_{\mathrm{rx}}$ similarly to (2) and (4), respectively. Then, the operator $\mathcal{I}_{\mathrm{rx}}$ is defined as

$$
\begin{aligned}
& \mathcal{I}_{\mathrm{rx}} s[n]=s[n]+\frac{c_{\mathrm{rx}, 2}}{c_{\mathrm{rx}, 1}^{*}} s^{*}[n]=s[n]+b_{\mathrm{rx}} s^{*}[n], \\
& \mathcal{I}_{\mathrm{rx}} S_{i}[k]=S_{i}[k]+\frac{c_{\mathrm{rx}, 2}}{c_{\mathrm{rx}, 1}^{*}} S_{i}^{*}[-k]=S_{i}[k]+b_{\mathrm{rx}} S_{i}^{*}[-k],
\end{aligned}
$$

where $b_{\mathrm{rx}}=c_{\mathrm{rx}, 2} / c_{\mathrm{rx}, 1}^{*}$. Thus, the received baseband signal can be expressed with the operator $\mathcal{I}_{\mathrm{rx}}$ as

$$
y[n]=\mathcal{I}_{\mathrm{rx}}\left(c_{\mathrm{rx}, 1} y_{\mathrm{LNA}}[n]\right),
$$

where $y_{\mathrm{LNA}}[n]$ is the output signal of the LNA.

\section{Nonlinear Distortions of Amplifiers}

The upconverted RF signal $x_{\mathrm{IQ}}[n]$ is amplified by the variable gain amplifier and the PA to radiate from the antenna subsequently. Similar to the IQ imbalance, the self-interference signal is distorted by the nonlinearity of the amplifiers, and it is expressed as [54, page 69]

$$
x_{\mathrm{PA}}[n]=\sum_{p=1,3, \cdots}^{\infty} a_{\mathrm{tx}, p} x_{\mathrm{IQ}}[n]\left|x_{\mathrm{IQ}}[n]\right|^{p-1},
$$

where $a_{\mathrm{tx}, p}$ is the gain of the $p$ th nonlinear distortion of the $\mathrm{PA}$, and $a_{\mathrm{tx}, 1}$ is the linear gain of transmitter RF amplifiers. To simplify the explanation in Section III, we define the operator $\mathcal{D}_{\text {tx }}$ for any input signal $s[n]$ as

$$
\mathcal{D}_{\mathrm{tx}} s[n]=s[n]+\sum_{p=3,5, \cdots}^{\infty} d_{\mathrm{tx}, p} s[n]|s[n]|^{p-1},
$$

where $d_{\mathrm{tx}, p}=\left|c_{\mathrm{tx}, 1}\right|^{p-1} a_{\mathrm{tx}, p} / a_{\mathrm{tx}, 1}$, and $d_{\mathrm{tx}, 1}$ is equal to 1. Then, the transmit signal from the transmit antenna is expressed as

$$
x_{\mathrm{PA}}[n]=a_{\mathrm{tx}, 1} c_{\mathrm{tx}, 1} \mathcal{D}_{\mathrm{tx}} \mathcal{I}_{\mathrm{tx}} x[n] .
$$

At the receiver, if the self-interference is strong enough to saturate the LNA, its nonlinear distortion will also occur from the LNA. The output signal of the LNA can be expressed as

$$
y_{\mathrm{LNA}}[n]=\sum_{p=1,3, \cdots}^{\infty} a_{\mathrm{rx}, p} y_{\mathrm{ASIC}}[n]\left|y_{\mathrm{ASIC}}[n]\right|^{p-1},
$$

where $y_{\mathrm{ASIC}}[n]$ is the signal after analog self-interference cancellation, and $a_{\mathrm{rx}, p}$ is the gain of the $p$ th distortion signal 
of the LNA. Similar to the PA distortion operator of (9), we define the operator $\mathcal{D}_{\text {rx }}$ as

$$
\mathcal{D}_{\mathrm{rx}} s[n]=s[n]+\sum_{p=3,5, \ldots}^{\infty} d_{\mathrm{rx}, p} s[n]|s[n]|^{p-1},
$$

where

$$
d_{\mathrm{rx}, p}=\frac{a_{\mathrm{rx}, p}}{a_{\mathrm{rx}, 1}\left|c_{\mathrm{rx}, 1} a_{\mathrm{rx}, 1}\right|^{p-1}} .
$$

Thus, the output signal of the received IQ mixer can be expressed as

$$
y[n]=\mathcal{I}_{\mathrm{rx}} \mathcal{D}_{\mathrm{rx}}\left(c_{\mathrm{rx}, 1} a_{\mathrm{rx}, 1} y_{\mathrm{ASIC}}[n]\right) .
$$

\section{SI Channel and RF Cancellation}

The TX and RX antennas are strongly coupled because terminals such as smartphones and IoT devices have limited size. Therefore, the self-interference channel can be modeled as a Rician fading channel with $20 \mathrm{~dB}$ or higher $\mathrm{K}$ factor [17]. Analog self-interference cancellation is used to reduce the received self-interference signal. Thus, the self-interference signal remaining after RF cancellation is expressed as

$$
\begin{aligned}
y_{\mathrm{ASIC}}[n] & =\left(h_{\mathrm{SI}}[m]-h_{\mathrm{Cir}}[m]\right) * x_{\mathrm{PA}}[n] \\
& =\sum_{m=0}^{M-1}\left(h_{\mathrm{SI}}[m]-h_{\mathrm{Cir}}[m]\right) x_{\mathrm{PA}}[n-m],
\end{aligned}
$$

where $h_{\mathrm{SI}}[m]$ is the wireless channel response, $h_{\mathrm{Cir}}[m]$ is the $\mathrm{RF}$ canceller circuits response, and $M$ is the number of taps of the wireless channel. To achieve high self-interference cancellation performance with an analog self-interference canceller, many variable attenuators and phase shifters are required, which increases the cost of the terminal. Thus, we assume that the RF canceller reduces only the direct wave of the selfinterference, and the channel response $h_{\mathrm{SI}}[m]-h_{\mathrm{Cir}}[m]$ can be modeled as a Rayleigh fading channel.

Then, we define the operator $\mathcal{H}$ as follows:

$$
\begin{aligned}
\mathcal{H} s[n] & =a_{\mathrm{tx}, 1} a_{\mathrm{rx}, 1} c_{\mathrm{tx}, 1} c_{\mathrm{rx}, 1}\left(h_{\mathrm{SI}}[m]-h_{\mathrm{Cir}}[m]\right) * s[n] \\
& =h[m] * s[n] \\
\mathcal{H} S_{i}[k] & =H[k] S_{i}[k]
\end{aligned}
$$

where $h[m]=a_{\mathrm{tx}, 1} a_{\mathrm{rx}, 1} c_{\mathrm{tx}, 1} c_{\mathrm{rx}, 1}\left(h_{\mathrm{SI}}[m]-h_{\mathrm{Cir}}[m]\right)$, and $H[k]$ is a frequency-domain representation of $h[m]$. To minimize the number of parameters for each operator, all amplification factors for linear SI components at the transmitter and receiver are aggregated into the operator $\mathcal{H}$. Then, the received SI signal $y[n]$ can be expressed simply as

$$
y[n]=\mathcal{I}_{\mathrm{rx}} \mathcal{D}_{\mathrm{rx}} \mathcal{H} \mathcal{D}_{\mathrm{tx}} \mathcal{I}_{\mathrm{tx}} x[n],
$$

Eq. (17) shows that the self-interference signal can be expressed concisely with operators and block diagrams as in Fig. 2. The operators $\mathcal{I}_{\mathrm{tx}}$ and $\mathcal{I}_{\mathrm{rx}}$ have only one parameter each, namely $b_{\mathrm{tx}}$ and $b_{\mathrm{rx}}$, respectively. In addition, the operators $\mathcal{D}_{\text {tx }}$ and $\mathcal{D}_{\text {rx }}$ have $(P-1) / 2$ parameters each if the nonlinear characteristic of an amplifier is approximated by a $P$-order polynomial. Thus, the total number of parameters of (17) is $P+M+1$, where $M$ is the number of taps of the channel impulse response $h[m]$.

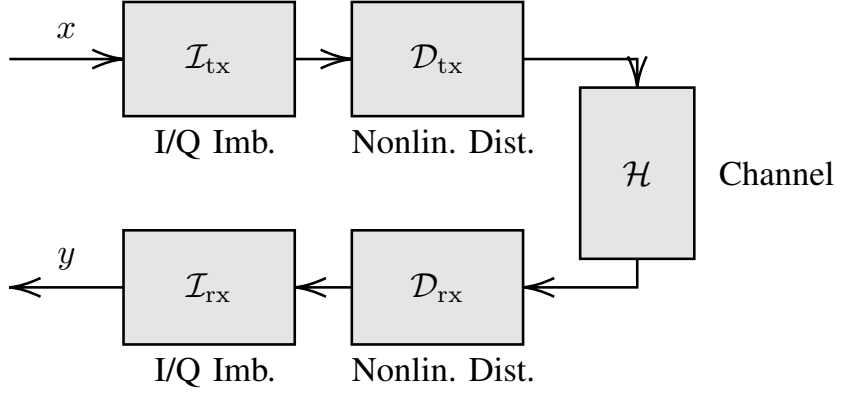

Fig. 2. Block diagram and signal flow of the self-interference with each RF component and operator

\section{Proposed Scheme}

In this section, we describe the proposed scheme, which consists of three estimation stages and a cancellation stage. The proposed scheme iteratively estimates the coefficients of IQ imbalance $b_{\mathrm{tx}}$ and $b_{\mathrm{rx}}$, the impulse response of the SI channel $h[m]$, and the nonlinear gains of amplifiers $d_{\mathrm{tx}, p}$ and $d_{\mathrm{rx}, p}$. In this section, $l$ denotes the iteration index of the estimation stage. On the $l$ th iteration, the proposed method updates the estimated values to more accurate ones using the values calculated at the $(l-1)$ th iteration.

\section{A. Definitions of Operators}

To explain the proposed scheme, we define the estimated value of each parameter on the $l$ th iteration as $\hat{b}_{\diamond, l}, \hat{h}_{l}[m]$, and $\hat{d}_{\diamond, p, l}$, where $\diamond \in\{\mathrm{tx}, \mathrm{rx}\}$. The initial values of these parameters are defined as

$$
\begin{aligned}
\hat{b}_{\diamond, 0} & =0, \\
\hat{d}_{\diamond, p, 0} & = \begin{cases}1 & \text { for } p=1, \\
0 & \text { for } p>1,\end{cases} \\
\hat{h}_{0}[m] & =\text { NaN for all } m,
\end{aligned}
$$

where $\mathrm{NaN}$ indicates that there is no initial value, and the proposed scheme does not need any initial values of $\hat{h}[\mathrm{~m}]$. Then, the estimated operators are defined using estimated parameters as

$$
\begin{aligned}
\hat{\mathcal{I}}_{\diamond, l} s[n] & =s[n]+\hat{b}_{\diamond, l} s^{*}[n], \\
\hat{\mathcal{D}}_{\diamond, l} s[n] & =s[n]+\sum_{p=3,5, \cdots}^{P} \hat{d}_{\diamond, p, l} s[n]|s[n]|^{p-1}, \\
\hat{\mathcal{H}}_{l} s[n] & =\sum_{m=0}^{M-1} \hat{h}_{l}[m] s[n-m], \\
\hat{\mathcal{H}}_{l} S_{i}[k] & =\hat{H}_{l}[k] S_{i}[k],
\end{aligned}
$$

where $P$ is the maximum estimation order of the nonlinearities, and $\hat{H}_{l}[k]$ is the frequency-domain representation of $\hat{h}_{l}[m]$. The inverse operator of $\hat{\mathcal{I}}_{\diamond, l}$ can be expressed as

$$
\hat{\mathcal{I}}_{\diamond, l}^{-1} s[n]=\frac{s[n]-\hat{b}_{\diamond, l} s^{*}[n]}{1-\left|\hat{b}_{\diamond, l}\right|^{2}} .
$$


The inverse operator of $\hat{\mathcal{D}}_{\diamond, l}$ is defined by Newton's method with $M_{\mathrm{n}}$ times iterations, and it can be expressed as

$$
\hat{\mathcal{D}}_{\diamond, l}^{-1} s[n]=D_{\diamond, l}^{-1}(|s[n]|) \frac{s[n]}{|s[n]|}
$$

where

$$
\begin{aligned}
D_{\diamond, l}^{-1}\left(r_{0}\right) & =r_{M_{\mathrm{n}}} \frac{\left|u_{M_{\mathrm{n}}}\right|}{u_{M_{\mathrm{n}}}}, \\
r_{k+1} & =r_{k}-\frac{\left(\left|u_{k}\right|-r_{0}\right)\left|u_{k}\right|}{\operatorname{Re}\left[u_{k}\right] \operatorname{Re}\left[v_{k}\right]+\operatorname{Im}\left[u_{k}\right] \operatorname{Im}\left[v_{k}\right]}, \\
u_{k} & =\hat{\mathcal{D}}_{\diamond, l} r_{k}, \\
v_{k} & =\sum_{p=1,3, \cdots}^{P} \hat{d}_{\diamond, p, l} p r_{k}^{p-1} .
\end{aligned}
$$

Detailed derivations of (26)-(30) are provided in Appendix A. We also define the operator $\mathcal{F}$, which denotes cyclic prefix (CP) removal and discrete Fourier transform for each OFDM symbol, and an operator $\mathcal{F}^{-1}$, which denotes the inverse discrete Fourier transform of each OFDM symbol.

\section{B. IQ Imbalance Estimation}

During the estimation of the IQ imbalance coefficients $b_{\text {tx }}$ and $b_{\mathrm{rx}}$, the other distortions, such as PA and LNA distortions, reduce the estimation accuracy. Thus, before estimating $b_{\mathrm{tx}}$ and $b_{\text {rx }}$ at the $l$ th iteration, we eliminate the distortion of the PA and LNA with the latest estimated values corresponding to the $(l-1)$ th iteration. In the proposed scheme, the transmitted and received signals for estimation are converted as follows:

$$
\begin{aligned}
& x_{l}^{(1)}[n]=\hat{\mathcal{I}}_{\mathrm{tx}, l-1}^{-1} \hat{\mathcal{D}}_{\mathrm{tx}, l-1} \hat{\mathcal{I}}_{\mathrm{tx}, l-1} x[n], \\
& y_{l}^{(1)}[n]=\hat{\mathcal{I}}_{\mathrm{rx}, l-1} \hat{\mathcal{D}}_{\mathrm{rx}, l-1}^{-1} \hat{\mathcal{I}}_{\mathrm{rx}, l-1}^{-1} y[n] .
\end{aligned}
$$

If the latest estimated values converge to the true values sufficiently, the relation of $x_{l}^{(1)}[n]$ and $y_{l}^{(1)}[n]$ is expressed as

$$
y_{l}^{(1)}[n]=\mathcal{I}_{\mathrm{rx}} \mathcal{H I}_{\mathrm{tx}} x_{l}^{(1)}[n]+z_{l}^{(1)}[n],
$$

where $z_{l}^{(1)}[n]$ is the error due to the estimation error of the latest estimated values. In (33), there is no distortion of the PA and LNA, and the error $z_{l}^{(1)}[n]$ can be decreased with each additional iteration. Thus, we can estimate the IQ imbalance coefficients $b_{\mathrm{tx}}$ and $b_{\mathrm{rx}}$ precisely from $x_{l}^{(1)}[n]$ and $y_{l}^{(1)}[n]$ when the latest estimated values have converged to the true values sufficiently. We use the frequency-domain representation of (31) and (32) to estimate the IQ imbalance coefficients. After CP removal and applying a discrete Fourier transform, we obtain the frequency representations $X_{i, l}^{(1)}[k]$ and $Y_{i, l}^{(1)}[k]$. The relation between these signal can be expressed as

$$
\begin{aligned}
Y_{i, l}^{(1)}[k] & =\underbrace{\left\{H[k]+b_{\mathrm{rx}} b_{\mathrm{tx}}^{*} H^{*}[-k]\right\}}_{H_{0, l}[k]} X_{i, l}^{(1)}[k] \\
& +\underbrace{\left\{b_{\mathrm{tx}} H[k]+b_{\mathrm{rx}} H^{*}[-k]\right\}}_{H_{1, l}[k]}\left(X_{i, l}^{(1)}[-k]\right)^{*}+Z_{i, l}^{(1)}[k] .
\end{aligned}
$$

Then, the channel frequency responses $H_{0}[k]$ and $H_{1}[k]$ are estimated by the well-known least squares method with $N_{\text {tr }}$ OFDM symbols at $-N_{\mathrm{sc}} \leq k \leq N_{\mathrm{sc}}$ and $k \neq 0$ as follows:

$$
\left[\begin{array}{c}
\hat{H}_{0, l}[k] \\
\hat{H}_{1, l}[k]
\end{array}\right]=\left\{\left(\mathbf{X}_{l}^{(1)}[k]\right)^{H} \mathbf{X}_{l}^{(1)}[k]\right\}^{-1}\left(\mathbf{X}_{l}^{(1)}[k]\right)^{H} \mathbf{Y}_{l}^{(1)}[k]
$$

where

$$
\begin{aligned}
& \mathbf{X}_{l}^{(1)}[k]=\left[\begin{array}{cccc}
X_{0, l}^{(1)}[k] & X_{1, l}^{(1)}[k] & \cdots & X_{N_{\mathrm{tr}}-1, l}^{(1)}[k] \\
\left(X_{0, l}^{(1)}[-k]\right)^{*} & \left(X_{1, l}^{(1)}[-k]\right)^{*} & \cdots & \left(X_{N_{\mathrm{tr}}-1, l}^{(1)}[-k]\right)^{*}
\end{array}\right]^{T}, \\
& \mathbf{Y}_{l}^{(1)}[k]=\left[Y_{0, l}^{(1)}[k] Y_{1, l}^{(1)}[k] \cdots Y_{N_{\mathrm{tr}}-1, l}^{(1)}[k]\right]^{T} .
\end{aligned}
$$

The estimated channel response $\hat{H}_{0, l}[k]$ can be well approximated to the self-interference channel response $H[k]$ because the value $\left|b_{\mathrm{rx}} b_{\mathrm{tx}}^{*}\right| \ll 1$ [55], [56]. Therefore, $H_{1, l}[k]$ is expressed as

$$
H_{1, l}[k] \approx b_{\mathrm{tx}} \hat{H}_{0, l}[k]+b_{\mathrm{rx}} \hat{H}_{0, l}^{*}[-k],
$$

and then we get the estimated values of the IQ imbalance coefficients as

$$
\left[\begin{array}{l}
\hat{b}_{\mathrm{tx}, l} \\
\hat{b}_{\mathrm{rx}, l}
\end{array}\right]=\left(\mathbf{H}_{0, l}^{H} \mathbf{H}_{0, l}\right)^{-1} \mathbf{H}_{0, l}^{H} \mathbf{H}_{1, l},
$$

where

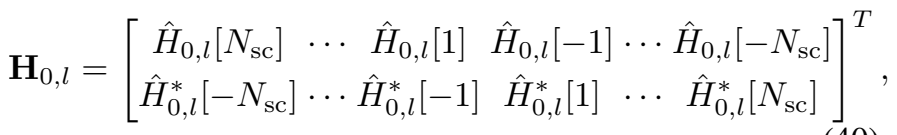

$\mathbf{H}_{1, l}=\left[\hat{H}_{1, l}\left[N_{\mathrm{sc}}\right] \cdots \hat{H}_{1, l}[1] \hat{H}_{1, l}[-1] \cdots \hat{H}_{1, l}\left[-N_{\mathrm{sc}}\right]\right]^{T}$.

\section{Channel Estimation}

After the IQ imbalance coefficients estimation stage, the proposed canceller estimates the channel impulse response $h[m]$. During the estimation of the channel impulse response, the nonlinear distortions of the IQ imbalance, PA, and LNA reduce the estimation accuracy of the channel impulse response. Thus, before estimating $h[m]$ at the $l$ th iteration, we eliminate the distortions with the latest estimated values. The transmitted and received signals for estimation are converted as follows:

$$
\begin{aligned}
& x_{l}^{(2)}[n]=\hat{\mathcal{D}}_{\mathrm{tx}, l-1} \hat{\mathcal{I}}_{\mathrm{tx}, l} x[n], \\
& y_{l}^{(2)}[n]=\hat{\mathcal{D}}_{\mathrm{rx}, l-1}^{-1} \hat{\mathcal{I}}_{\mathrm{rx}, l}^{-1} y[n] .
\end{aligned}
$$

If the latest estimated values converge to the true values sufficiently, the relation of $x_{l}^{(2)}[n]$ and $y_{l}^{(2)}[n]$ is expressed as

$$
\begin{aligned}
y_{l}^{(2)}[n] & =\mathcal{H} x_{l}^{(2)}[n]+z_{l}^{(2)}[n] \\
& =\sum_{m=0}^{M-1} h[m] x_{l}^{(2)}[n-m]+z_{l}^{(2)}[n],
\end{aligned}
$$

where $z_{l}^{(2)}[n]$ is the error caused by the estimation error of the latest estimated values. Then, the channel impulse response 


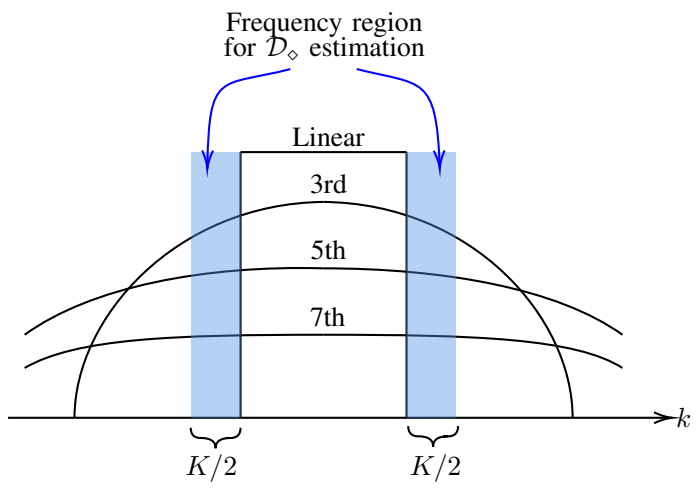

Fig. 3. Frequency region to estimate $d_{\diamond, p}$.

$h[m]$ is estimated by the well-known least-squares method with $N_{\text {tr }}$ OFDM symbols as

$$
\left[\begin{array}{c}
\hat{h}_{l}[0] \\
\vdots \\
\hat{h}_{l}[M-1]
\end{array}\right]=\underbrace{\left\{\left(\mathbf{X}_{l}^{(2)}\right)^{H} \mathbf{X}_{l}^{(2)}\right\}^{-1}}_{\mathbf{T}_{l}^{-1}}\left(\mathbf{X}_{l}^{(2)}\right)^{H} \mathbf{y}_{l}^{(2)}
$$

where $\mathbf{X}_{l}^{(2)}$ is the $\left(M+N_{\text {tr }} N_{\text {sym }}-1\right) \times M$ matrix whose $i$ th row and $j$ th column element are

$$
\mathbf{X}_{l}^{(2)}[i, j]= \begin{cases}0 & \text { if } i-j<0 \\ x_{l}^{(2)}[i-j] & \text { if } 0 \leq i-j<N_{\mathrm{tr}} N_{\mathrm{sym}}, \\ 0 & \text { if } N_{\mathrm{tr}} N_{\mathrm{sym}} \leq i-j\end{cases}
$$

and

$$
\mathbf{y}_{l}^{(2)}=\left[y_{l}^{(2)}[0] y_{l}^{(2)}[1] \cdots y_{l}^{(2)}\left[M+N_{\mathrm{tr}} N_{\mathrm{sym}}-2\right]\right],
$$

and $N_{\text {sym }}$ is the size of an OFDM symbol. In (45), $\mathbf{T}_{l}$ is a Hermitian Toeplitz matrix [57], and fast algorithms to solve (45) with $\mathcal{O}\left(M N_{\mathrm{tr}} N_{\mathrm{sym}}+M^{2}\right)$ multiplications and additions exist [58].

\section{Amplifier Nonlinearity Estimation}

After the channel estimation stage, we estimate the nonlinear coefficients of the amplifiers. To estimate coefficients $d_{\mathrm{tx}, p}$ and $d_{\mathrm{rx}, p}$, we need to eliminate the effects of IQ imbalance to obtain a high accuracy result. After applying the operator $\mathcal{I}_{\mathrm{rx}}^{-1}$ to both sides of (17) and some manipulations, we obtain the following equation:

$$
\begin{aligned}
\mathcal{I}_{\mathrm{rx}}^{-1} y[n]= & \mathcal{H} \mathcal{I}_{\mathrm{tx}} x[n]+\mathcal{H}\left(\mathcal{D}_{\mathrm{tx}}-1\right) \mathcal{I}_{\mathrm{tx}} x[n] \\
& +\left(\mathcal{D}_{\mathrm{rx}}-1\right) \mathcal{H} \mathcal{D}_{\mathrm{tx}} \mathcal{I}_{\mathrm{tx}} x[n] .
\end{aligned}
$$

In (48), $\mathcal{H} \mathcal{I}_{\mathrm{tx}} x[n]$ denotes the linear component of the selfinterference signal; $\mathcal{H}\left(\mathcal{D}_{\mathrm{tx}}-1\right) \mathcal{I}_{\mathrm{tx}} x[n]$ is caused by the TX amplifier, and $\left(\mathcal{D}_{\mathrm{rx}}-1\right) \mathcal{H} \mathcal{D}_{\mathrm{tx}} \mathcal{I}_{\mathrm{tx}} x[n]$ is caused by the TX and RX amplifiers. When estimating the nonlinear distortion coefficient, the signal of the linear component degrades the accuracy of the result. Thus, we need to eliminate the linear component from the received signal. In the discrete-frequency domain, when the frequency index $k$ indicates the sidelobe of the OFDM signal, such as guard bands, the linear component of the self-interference signal is equal to zero. Therefore, when $|k|>N_{\text {sc }} / 2$, we can write the following equation on the $i$ th OFDM symbol:

$$
\begin{aligned}
Y_{i, l}^{(3)}[k]= & \sum_{p=3,5, \cdots}^{P} d_{\mathrm{tx}, p} \Psi_{\mathrm{tx}, p, i, l}^{(3)}[k] \\
& +\sum_{p=3,5, \cdots}^{P} d_{\mathrm{rx}, p} \Psi_{\mathrm{rx}, p, i, l}^{(3)}[k]+Z_{i, l}^{(3)}[k],
\end{aligned}
$$

where

$$
\begin{aligned}
Y_{i, l}^{(3)}[k] & =\mathcal{F}_{\hat{\mathcal{I}}_{\mathrm{rx}, l}^{-1} y[n],} \\
x_{l}^{(3)}[n] & =\hat{\mathcal{I}}_{\mathrm{tx}, l} x[n], \\
u_{l}^{(3)}[n] & =\mathcal{F}^{-1} \hat{\mathcal{H}}_{l} \mathcal{F} \hat{\mathcal{D}}_{\mathrm{tx}, l} x_{l}^{(3)}[n], \\
\Psi_{\mathrm{tx}, p, i, l}^{(3)}[k] & =\hat{\mathcal{H}}_{l} \mathcal{F}\left(x_{l}^{(3)}[n]\left|x_{l}^{(3)}[n]\right|^{p-1}\right), \\
\Psi_{\mathrm{rx}, p, i, l}^{(3)}[k] & =\mathcal{F}\left(u_{l}^{(3)}[n]\left|u_{l}^{(3)}[n]\right|^{p-1}\right),
\end{aligned}
$$

and $Z_{i, l}^{(3)}[k]$ is the error caused by the estimation error of the latest estimated values. Thus, we can estimate the coefficients of the nonlinearities with $K$ sidelobe subcarriers by

$$
\hat{\mathbf{d}}_{l}=\left\{\left(\boldsymbol{\Psi}_{l}^{(3)}\right)^{H} \boldsymbol{\Psi}_{l}^{(3)}\right\}^{-1}\left(\boldsymbol{\Psi}_{l}^{(3)}\right)^{H} \mathbf{Y}_{l}^{(3)},
$$

where

$$
\begin{aligned}
& \hat{\mathbf{d}}_{l}=\left[\begin{array}{llllll}
\hat{d}_{\mathrm{tx}, 3, l} & \cdots & \hat{d}_{\mathrm{tx}, P, l} & \hat{d}_{\mathrm{rx}, 3, l} & \cdots & \hat{d}_{\mathrm{rx}, P, l}
\end{array}\right]^{T}, \\
& \mathbf{\Psi}_{l}^{(3)}=\left[\left(\mathbf{\Psi}_{0, l}^{(3)}\right)^{T}\left(\mathbf{\Psi}_{1, l}^{(3)}\right)^{T} \cdots\left(\mathbf{\Psi}_{N_{\mathrm{tr}}-1, l}^{(3)}\right)^{T}\right]^{T},
\end{aligned}
$$

$\mathbf{Y}_{l}^{(3)}=\left[\begin{array}{lllll}\mathbf{Y}_{0, l}^{(3)} & \mathbf{Y}_{1, l}^{(3)} & \cdots & \mathbf{Y}_{N_{\mathrm{tr}}-1, l}^{(3)}\end{array}\right]^{T}$,

$\mathbf{Y}_{i, l}^{(3)}=\left[Y_{i, l}^{(3)}\left[k_{1}\right] Y_{i, l}^{(3)}\left[k_{2}\right] \cdots Y_{i, l}^{(3)}\left[k_{K}\right]\right]$,

and $k_{1}, k_{2}, \cdots, k_{K}$ are selected from the discrete-frequency of sidelobe, as shown in Fig. 3.

\section{E. Self-Interference Cancellation}

After some iterations, the canceller cancels the selfinterference from the received signal. The received signal, which includes the desired signal from another terminal, is also distorted by the RX amplifier and the IQ mixer imbalance. Thus, the best cancellation approach is nonlinear signal reconstruction and cancellation with post-distortion, which gives the received signal the inverse of the RX distortion. In the proposed scheme, we have the inverse operators $\hat{\mathcal{I}}_{\mathrm{rx}, L}^{-1}$ and $\hat{\mathcal{D}}_{\mathrm{rx}, L}^{-1}$, which can be used for the post-distortion. Therefore, the signal after cancellation is

$$
y_{\mathrm{DC}}[n]=\hat{\mathcal{D}}_{\mathrm{rx}, L}^{-1} \hat{\mathcal{I}}_{\mathrm{rx}, L}^{-1} y[n]-\hat{\mathcal{H}}_{L} \hat{\mathcal{D}}_{\mathrm{tx}, L} \hat{\mathcal{I}}_{\mathrm{tx}, L} x[n] .
$$




\section{F. Pseudo Code and Computational Cost}

TABLE II lists the computational cost of each operator. In addition, TABLE III, TABLE IV, and TABLE V show the computation steps and computational costs of the estimation stages of IQ imbalance, channel, and nonlinearity, respectively. In these tables, $N_{\mathrm{fft}}$ is the number of samples of an OFDM symbol after CP removal, and "MULs/DIVs" and "ADDs/SUBs" denote "multiplications and divisions" and "additions and subtractions," respectively. In the cancellation stage, the proposed scheme computes (61), and needs the following computational cost:

- \# of MULs/DIVs per OFDM symbol is $N_{\text {sym }}\left(M+M_{\mathrm{n}}(1.5 P+5.5)+\frac{1}{2} P+6.5\right)$.

- \# of ADDs/SUBs per OFDM symbol is $N_{\text {sym }}\left(M+M_{\mathrm{n}}(P+2)+\frac{1}{2} P+3.5\right)$.

- \# of $\sqrt{x}$ per OFDM symbol is $N_{\mathrm{sym}}\left(M_{\mathrm{n}}+2\right)$.

\section{Numerical Simulations}

\section{A. Simulation Environment}

We perform equivalent baseband simulations of the fullduplex transceiver to verify the proposed estimation and cancellation scheme. TABLE VI and TABLE VII list the parameters of the simulations. The baseband signal simulator is implemented with $\mathrm{D}$ programming language, and the details of the simulator are as follows.

In the simulations, the self-interference channel, which consists of the wireless multipath channel and the impulse response of the RF self-interference canceller, is modeled as a quasi-static Rayleigh fading channel with a constant impulse response on a simulation trial and has different impulse responses between different simulations trials. The power delay profile of the self-interference channel is modeled as an exponential decay profile with $40 \mathrm{~dB}$ decay at 48 delay samples, and each tap of the channel impulse response is independent and identically distributed on the complex normal distribution.

The most severe bottleneck for self-interference cancellation is the non-idealities of the IQ mixers, PA, and LNA. The simulation model of IQ mixers achieves IQ imbalance by adding an image signal, and its coefficients are predetermined based on the value of IRR. The nonlinearity of the PA and LNA is characterized by the Rapp model [60], which is often used to simulate the baseband behaviors of class AB solid-state amplifiers. The output baseband signal of a Rapp modeled amplifier is described as

$$
v_{\text {out }}=\frac{G v_{\text {in }}}{\left[1+\left(\frac{\left|v_{\text {in }}\right|}{V_{\text {sat }}}\right)^{2 s}\right]^{\frac{1}{2 s}}},
$$

where $v_{\text {in }}$ and $v_{\text {out }}$ are the input and output signals of the amplifier, respectively; $G, V_{\text {sat }}$, and $s$ are the gain, saturated input level, and smoothness factor of the Rapp model, respectively, and they characterize the nonlinearity of the amplifier.

To cancel nonlinearities up to seventh order, the maximum order of the nonlinear coefficients estimation, which is a parameter of the operator $\hat{\mathcal{D}}_{\diamond, l}$ in (22), is $P=7$ for

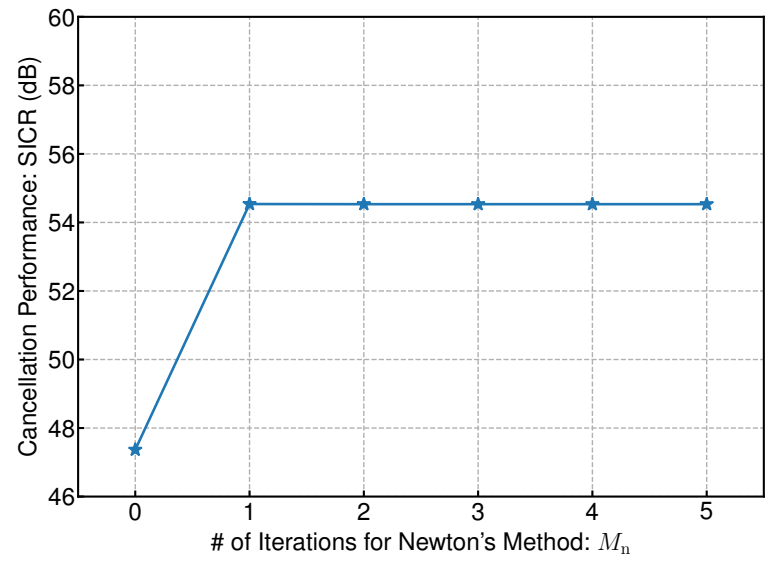

Fig. 4. Performance of the proposed canceller with different numbers of iterations of Newton's method. The INR is $60 \mathrm{~dB}$, and the proposed canceller estimates parameters in ten iterations. The canceller is trained with 20 OFDM symbols.

the proposed canceller. Furthermore, the number of taps of channel estimation, which is a parameter of the operator $\hat{\mathcal{H}}_{l}$ in (23), is $M=48$.

Following the standard convention, we define the selfinterference cancellation ratio (SICR), which indicates the performance of a digital canceller, as

$$
\mathrm{SICR}=\frac{\mathbb{E}\left[|y[n]|^{2}\right]}{\mathbb{E}\left[\left|y_{\mathrm{DC}}[n]\right|^{2}\right]} .
$$

In addition, we define the amount of attenuation of the antenna separation and the RF self-interference cancellation as

$$
A_{\mathrm{RF}}=\frac{\mathbb{E}\left[\left|x_{\mathrm{PA}}[n]\right|^{2}\right]}{\mathbb{E}\left[\left|h_{\mathrm{SI}}[m] * x_{\mathrm{PA}}[n]-h_{\mathrm{Cir}}[m] * x_{\mathrm{PA}}[n]\right|^{2}\right]} .
$$

Then, the self-interference to noise power ratio (INR) is defined as

INR $(\mathrm{dB})=$ Transmission Power $(\mathrm{dBm})-$ AWGN Power $(\mathrm{dBm})$

$$
\text { -LNA Noise Figure }(\mathrm{dB})-A_{\mathrm{RF}}(\mathrm{dB}) \text {, }
$$

which indicates the theoretical limit of the SICR when the LNA is not saturated.

\section{B. Results and Discussions}

Fig. 4 shows the performance of the proposed canceller with different numbers of iterations of Newton's method. In this result, the received signal saturates the LNA, and it is highly distorted. If the number of iterations is zero, Newton's method outputs the input signal without any changes. Thus, the estimated coefficients of the LNA nonlinearity are not used effectively for the next iteration, and the cancellation performance of the proposed scheme is degraded. When the number of the iteration is one, the cancellation performance is saturated at approximately $54 \mathrm{~dB}$. Therefore, in the rest of this paper, the number of Newton's method iterations is set as $M_{\mathrm{n}}=1$. 
TABLE II

Computational Cost of Primitive Operations with $n$-Length Signal

\begin{tabular}{c|c|c|c|c}
\hline \hline Operation & Defined by & MULs/DIVs & ADDs/SUBs & $\sqrt{x}$ \\
\hline$\hat{\mathcal{I}}_{\diamond, l} s[i]$ & $(21)$ & $n$ & $n$ & 0 \\
\hline$\hat{\mathcal{I}}_{\diamond, l}^{-1} s[i]$ & $(25)$ & $3 n$ & $2 n$ & 0 \\
\hline$\hat{\mathcal{H}}_{l} s[i]$ & $(23)$ & $M n$ & $M n$ & 0 \\
\hline$\hat{\mathcal{H}}_{l} S[k]$ & $(24)$ & $n$ & $n$ & 0 \\
\hline$\hat{\mathcal{D}}_{\diamond, l} s[i]$ with Horner's algorithm & $(22)$ & $\frac{1}{2}(P+1) n$ & $\frac{1}{2}(P-1) n$ & $n\left(M_{\mathrm{n}}+2\right)$ \\
\hline$\hat{\mathcal{D}}_{\diamond, l}^{-1} s[i]$ & $(26)$ & $n M_{\mathrm{n}}(P+2)$ & $n M_{\mathrm{n}}(1.5 P+5.5)+2 n$ & 0 \\
\hline Solve triangular system [58, sec. 5.4] & - & $\frac{1}{2} n^{2}$ & $\frac{1}{2} n^{2}$ & 0 \\
\hline $\begin{array}{c}\text { Solve Toeplitz system } \\
\text { by Schur algorithm [58, sec. 5.4] }\end{array}$ & - & $3 n^{2}$ & $3 n^{2}$ & $n$ \\
\hline Cholesky decomposition [59] & - & $\frac{1}{6} n^{3}+\frac{1}{2} n^{2}-\frac{5}{3} n+1$ & $\frac{1}{6} n^{3}-\frac{1}{6} n$ & $n$ \\
\hline $\begin{array}{c}\text { Solve Hermite system } \\
\text { with Cholesky decomposition }\end{array}$ & - & $\frac{1}{6} n^{3}+\frac{3}{2} n^{2}-\frac{5}{3} n+1$ & $\frac{1}{6} n^{3}+n^{2}-\frac{1}{6} n$ & $n$ \\
\hline \hline
\end{tabular}

TABLE III

Pseudo Code and Computational Cost of IQI Estimation Stage

\begin{tabular}{l|c|c|c}
\hline \hline Step & MULs/DIVs & ADDs/SUBs & $\sqrt{x}$ \\
\hline$x_{l}^{(1)}[n] \leftarrow(31)$ & $N_{\mathrm{sym}} N_{\mathrm{tr}}\left(\frac{1}{2} P+4.5\right)$ & $N_{\mathrm{sym}} N_{\mathrm{tr}}\left(\frac{1}{2} P+2.5\right)$ & 0 \\
$y_{l}^{(1)}[n] \leftarrow(32)$ & $N_{\mathrm{sym}} N_{\mathrm{tr}}\left(M_{\mathrm{n}}(1.5 P+5.5)+6\right)$ & $N_{\mathrm{sym}} N_{\mathrm{tr}}\left(M_{\mathrm{n}}(P+2)+3\right)$ & $N_{\mathrm{sym}} N_{\mathrm{tr}}\left(M_{\mathrm{n}}+2\right)$ \\
Apply FFT to each symbol of $x_{l}^{(1)}[n]$ & $\frac{1}{2} N_{\mathrm{fft}} N_{\mathrm{tr}} \log _{2} N_{\mathrm{fft}}$ & $N_{\mathrm{fft}} N_{\mathrm{tr}} \log _{2} N_{\mathrm{fft}}$ & 0 \\
Apply FFT to each symbol of $y_{l}^{(1)}[n]$ & $\frac{1}{2} N_{\mathrm{fft}} N_{\mathrm{tr}} \log _{2} N_{\mathrm{fft}}$ & $N_{\mathrm{fft}} N_{\mathrm{tr}} \log _{2} N_{\mathrm{fft}}$ & 0 \\
Solve LS of (35) for all $k$ & $N_{\mathrm{sc}}\left(6 N_{\mathrm{tr}}+5\right)$ & $N_{\mathrm{sc}}\left(6 N_{\mathrm{tr}}+5\right)$ & $2 N_{\mathrm{sc}}$ \\
Solve LS of (39) & $6 N_{\mathrm{sc}}+5$ & $6 N_{\mathrm{sc}}+5$ & 2 \\
\hline \hline
\end{tabular}

TABLE IV

Pseudo Code and Computational Cost of Channel Estimation Stage

\begin{tabular}{l|c|c|c}
\hline \hline Step & MULs/DIVs & ADDs/SUBs & $\sqrt{x}$ \\
\hline$x_{l}^{(2)}[n] \leftarrow(42)$ & $N_{\mathrm{sym}} N_{\mathrm{tr}}\left(\frac{1}{2} P+1.5\right)$ & $\frac{1}{2} N_{\mathrm{sym}} N_{\mathrm{tr}}(P+1)$ & 0 \\
$y_{l}^{(2)}[n] \leftarrow(43)$ & $N_{\mathrm{sym}} N_{\mathrm{tr}}\left(M_{\mathrm{n}}(1.5 P+5.5)+5\right)$ & $N_{\mathrm{sym}} N_{\mathrm{tr}}\left(M_{\mathrm{n}}(P+2)+2\right)$ & $N_{\mathrm{sym}} N_{\mathrm{tr}}\left(M_{\mathrm{n}}+2\right)$ \\
Compute $\mathbf{T}_{l}=\left(\mathbf{X}_{l}^{(2)}\right)^{H} \mathbf{X}_{l}^{(2)}$ & $M\left(\frac{1}{2} M+N_{\mathrm{sym}} N_{\mathrm{tr}}-\frac{1}{2}\right)$ & $M\left(\frac{1}{2} M+N_{\mathrm{sym}} N_{\mathrm{tr}}-\frac{1}{2}\right)$ & 0 \\
Compute $\mathbf{u}_{l}=\left(\mathbf{X}_{l}^{(2)}\right)^{H} \mathbf{y}_{l}^{(2)}$ & $M\left(M+N_{\mathrm{sym}} N_{\mathrm{tr}}-1\right)$ & $M\left(M+N_{\mathrm{sym}} N_{\mathrm{tr}}-1\right)$ & 0 \\
Solve $\mathbf{T}_{l} \hat{\mathbf{h}}_{l}=\mathbf{u}_{l}$ & $3 M^{2}$ & $3 M^{2}$ & 0 \\
\hline \hline
\end{tabular}

TABLE V

Pseudo Code and Computational Cost of Nonlinear Estimation Stage

\begin{tabular}{l|c|c|c}
\hline \hline Step & MULs/DIVs & ADDs/SUBs & $\sqrt{x}$ \\
\hline apply FFT to $\hat{h}_{l}[m]$ & $\frac{1}{2} N_{\mathrm{fft}} \log _{2} N_{\mathrm{fft}}$ & $N_{\mathrm{fft}} \log _{2} N_{\mathrm{fft}}$ & 0 \\
$Y_{i, l}^{(3)}[k] \leftarrow(50)$ & $N_{\mathrm{tr}}\left(\frac{1}{2} N_{\mathrm{fft}} \log _{2} N_{\mathrm{fft}}+3 N_{\mathrm{sym}}\right)$ & $N_{\mathrm{tr}}\left(N_{\mathrm{fft}} \log _{2} N_{\mathrm{fft}}+2 N_{\mathrm{sym}}\right)$ & 0 \\
$x_{l}^{(3)}[n] \leftarrow(51)$ & $N_{\mathrm{fft}} N_{\mathrm{tr}}$ & $N_{\mathrm{fft}} N_{\mathrm{tr}}$ & 0 \\
$u_{l}^{(3)}[n] \leftarrow(52)$ & $N_{\mathrm{fft}} N_{\mathrm{tr}}\left(\frac{1}{2} P+\log _{2} N_{\mathrm{fft}}+1.5\right)$ & $N_{\mathrm{fft}} N_{\mathrm{tr}}\left(\frac{1}{2} P+2 \log _{2} N_{\mathrm{fft}}-\frac{1}{2}\right)$ & 0 \\
$\Psi_{\mathrm{tx}, p, i, l}^{(3)}[k] \leftarrow(53)$ & $\frac{1}{2} N_{\mathrm{tr}}\left(N_{\mathrm{fft}}(P+1)+\left(K+\frac{1}{2} N_{\mathrm{fft}} \log _{2} N_{\mathrm{fft}}\right)(P-1)\right)$ & $\frac{1}{2} N_{\mathrm{fft}} N_{\mathrm{tr}}(P-1) \log _{2} N_{\mathrm{fft}}$ & 0 \\
$\Psi_{\mathrm{rx}, p, i, l}^{(3)}[k] \leftarrow(54)$ & $N_{\mathrm{fft}} N_{\mathrm{tr}}\left(\frac{1}{2} P+\frac{1}{4}(P-1) \log _{2} N_{\mathrm{fft}}+\frac{1}{2}\right)$ & $\frac{1}{2} N_{\mathrm{fft}} N_{\mathrm{tr}}(P-1) \log _{2} N_{\mathrm{fft}}$ & 0 \\
Solve LS of (55) & $K N_{\mathrm{tr}} P(P-1)+\frac{P^{3}}{6}+P^{2}-\frac{25}{6} P+4$ & $K N_{\mathrm{tr}} P(P-1)+\frac{P^{3}}{6}+\frac{P^{2}}{2}-\frac{5 P}{3}+1$ & $P-1$ \\
\hline \hline
\end{tabular}

In Fig. 5, the performance of the proposed canceller is shown with different numbers of iterations of the estimation process in the same condition as that in Fig. 4. Labels, e.g. "IHD", indicate the order of estimation stages, such as $\mathcal{I}_{\diamond}, \mathcal{H}$, and $\mathcal{D}_{\diamond}$, and "IHD" indicates the order described in Section III. When the number of iterations is one, the proposed scheme does not use the first estimated parameters to improve the estimation quality. Thus, the cancellation performance with the single estimation iteration is approximately $22 \mathrm{~dB}$ lower than the saturated performance. If the number of iterations increases from one to two, the performance of the proposed scheme is significantly improved. Moreover, "IHD" achieves the best performance over the other orders. The reason is that the estimation stage of the imbalance coefficients does not depend on the estimated value of channel impulse response, and the number of these parameters is only two. In other words, the stage which is easiest to estimate is the estimation of the IQ imbalance coefficients. Therefore, we applied "IHD"- 
TABLE VI

OFDM MODULATION SPECIFICATIONS

\begin{tabular}{c|c}
\hline \hline Parameter & Value \\
\hline Modulation & OFDM \\
\hline Constellation & 16QAM \\
\hline FFT size & 64 \\
\hline Active subcarriers & 52 \\
\hline Cyclic prefix size & 16 \\
\hline Bandwidth & $20 \mathrm{MHz}$ \\
\hline \hline
\end{tabular}

TABLE VII

SIMULATION SPECIFICATIONS

\begin{tabular}{c|c}
\hline \hline Parameter & Value \\
\hline Oversampling rate & 8 \\
\hline Sampling rate & $20 \mathrm{MHz} \times 8=160 \mathrm{MHz}$ \\
\hline SI channel after RF-SIC & Rayleigh fading \\
\hline SI channel length & 48 samples \\
\hline Channel profile & Exponential decay \\
\hline IRR & $25 \mathrm{~dB}$ \\
\hline Transmission power & $33 \mathrm{dBm}$ \\
\hline PA Gain & $30 \mathrm{~dB}$ \\
\hline PA input saturation level & $($ IBO $=7 \mathrm{~dB}$ @ Transmission power $=23 \mathrm{dBm}$ ) \\
\hline PA smoothness factor & 3 \\
\hline LNA noise figure & $4 \mathrm{~dB}$ \\
\hline LNA Gain & $20 \mathrm{~dB}$ \\
\hline LNA input saturation level & $\left.V_{\text {sat,LNA }=-6 \mathrm{dBm}}=0.6 \mathrm{dBm}\right)$ \\
\hline LNA smoothness factor & 1 \\
\hline \# of ADC bits & 14 bit \\
\hline Order of canceller & $P=7$ \\
\hline \# of taps of canceller & $M=48$ \\
\hline Trials & 201 \\
\hline \hline
\end{tabular}

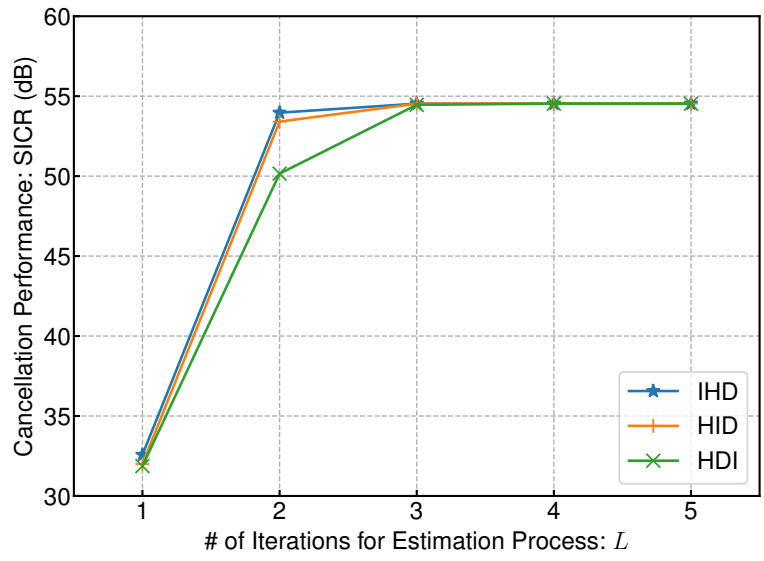

Fig. 5. Performance of the proposed canceller with different numbers of iterative estimation and different orders of estimation. The INR is $60 \mathrm{~dB}$, and the number of iterations of Newton's method is one. The canceller is trained with 20 OFDM symbols.

order and three-time iterations for the parameter estimation process because the proposed scheme can achieve sufficiently high cancellation performance with these settings.

Fig. 6 shows the performance of the proposed canceller with different values of $K$, which is the number of sidelobe subcarriers for nonlinear coefficients estimations. The performance difference between $K=2$ and $K=20$ is less than $1 \mathrm{~dB}$. Thus, there is no significant performance degradation, even if

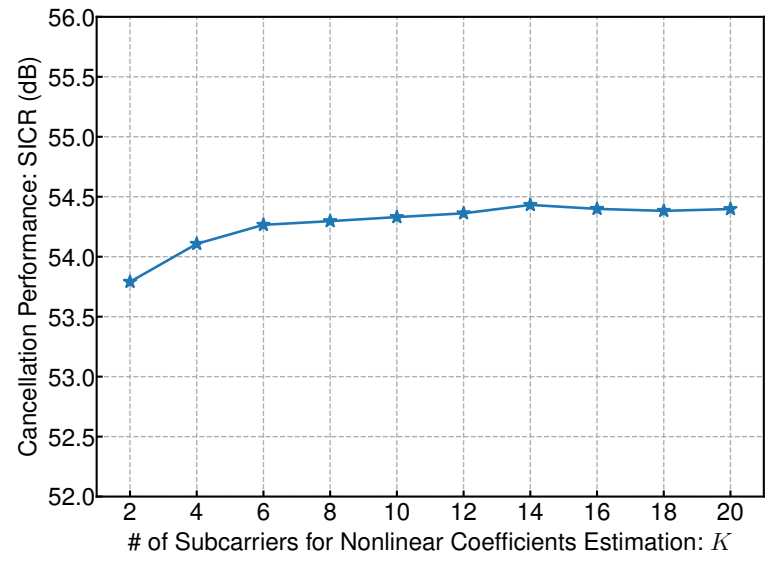

Fig. 6. Performance of the proposed canceller with different numbers of subcarriers for estimation of nonlinear coefficients. The INR is $60 \mathrm{~dB}$; the number of iterations of Newton's method is one; the number of iterations of the estimation process is three. The canceller is trained with 20 OFDM symbols.

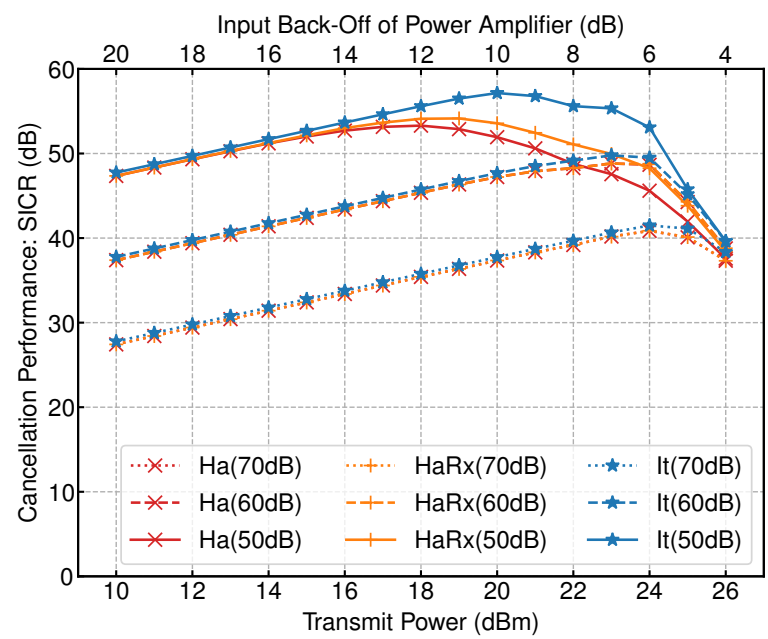

Fig. 7. Performance of each canceller with 100 training symbols on different amounts of RF attenuation $A_{\mathrm{RF}}$ and different transmission powers. The numbers in parentheses in the legend denote the values of $A_{\mathrm{RF}}$. In the proposed scheme, $M_{\mathrm{n}}=1, L=3$, and $K=8$.

only a small number of sidelobe subcarriers can be used for estimation. Besides, the performance with $K=8$ or more is almost the same as that with $K=20$, and it is possible to estimate by using the guard band. Therefore, in the rest of this section, we use eight sidelobe subcarriers $(K=8)$ to estimate nonlinear coefficients.

In Fig. 7, we compare the performance of the proposed canceller (denoted as "It") and a Hammerstein canceller with 20 basis functions (denoted as "Ha") that can estimate and regenerate seventh-order PA nonlinearities and a 48-samplesdelayed signal correctly. To make fair comparisons with the proposed canceller, the LS algorithm is used as the estimation algorithm for the Hammerstein canceller. For comparison with other methods that consider LNA nonlinearity, we also show the performance of a canceller (denoted as "HaRx") 


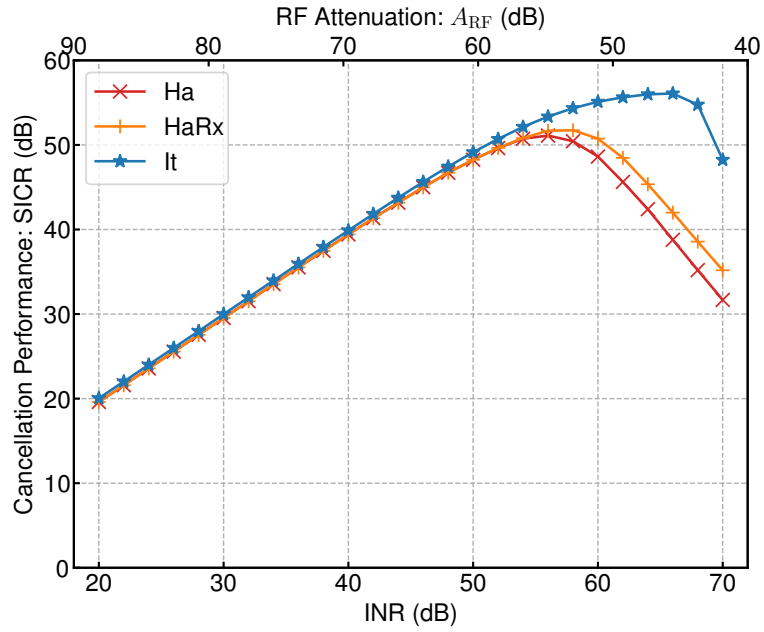

Fig. 8. Performance of each canceller with 100 training symbols and different self-interference powers. In this result, $M_{\mathrm{n}}=1, L=3$, and $K=8$.

based on the technique described in [44]. Because the RX nonlinearity mitigation technique presented in [44] is designed for wideband receivers, the HaRx canceller combines the technique described in [44] and the Hammerstein canceller. In Fig. 7, we give 100 training OFDM symbols to each canceller to compare their best performance. If the RF attenuation $A_{\mathrm{RF}}$, which contains antenna separation and RF circuit cancellation, is larger than $60 \mathrm{~dB}$, the performance of the three cancellers is almost the same. Additionally, if $A_{\mathrm{RF}}$ is $50 \mathrm{~dB}$, the proposed canceller and the HaRx canceller achieve higher performance than the Hammerstein canceller when the transmission power is higher than $17 \mathrm{dBm}$. In this region, the received signal is larger than $-33 \mathrm{dBm}$, and the nonlinear distortion of the LNA becomes larger than the thermal noise level. The Hammerstein canceller cannot reduce the nonlinearity of the LNA, and its performance decreases significantly as the received power increases. In contrast, the HaRx canceller and the proposed canceller can mitigate the received self-interference signal distorted by the LNA. However, the HaRx canceller only improves the cancellation performance by a few $\mathrm{dB}$ because it is just a simple combination of the Hammerstein canceller and technique reported in [44], and each of its components operates independently. The proposed canceller achieves a better performance improvement than the HaRx canceller because parameters are estimated in cooperation with the three stages.

For a more detailed discussion, we compare the cancellers in Fig. 8, which shows the canceller performance when the INR is changed from 20 to $70 \mathrm{~dB}$. Because the power of the received self-interference signal increases as the INR increases, the nonlinearity of the LNA increases as the INR increases. When the INR is larger than approximately 52 $\mathrm{dB}$, the performance of the Hammerstein canceller reaches $51 \mathrm{~dB}$ because it cannot regenerate the nonlinearity of the LNA. Fig. 8 shows that the proposed canceller achieves a performance up to $20 \mathrm{~dB}$ higher than that of the Hammerstein canceller, which indicates that the proposed canceller can

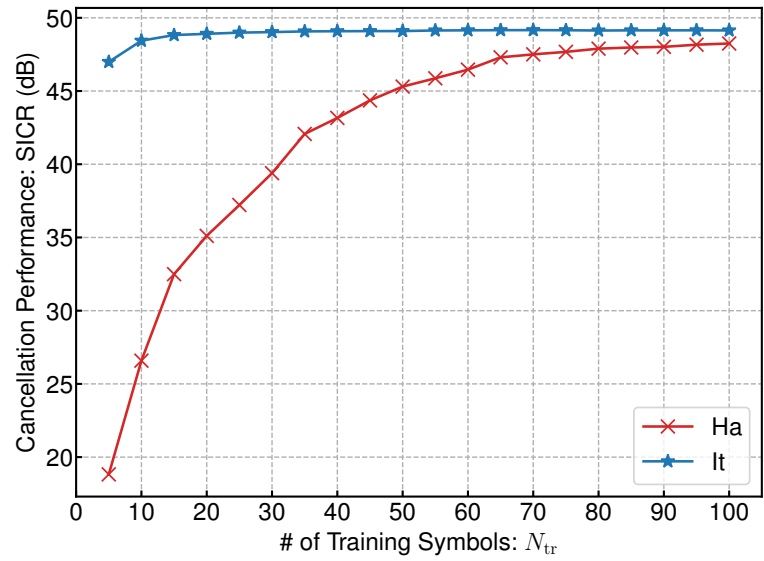

Fig. 9. Convergence of each canceller at INR $=50 \mathrm{~dB}$. In this result, $M_{\mathrm{n}}=1$, $L=3$, and $K=8$.

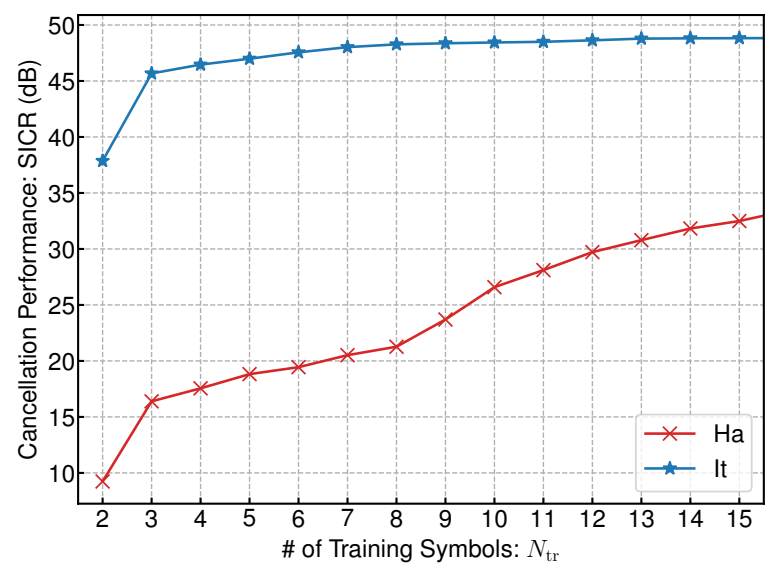

Fig. 10. Short span convergence of each canceller at INR $=50 \mathrm{~dB}$. In this result, $M_{\mathrm{n}}=1, L=3$, and $K=8$.

apply the inverse of the LNA nonlinearity to the received signal and endure higher power of self-interference.

To discuss the convergence speed, we consider the results shown in Fig. 9 and 10. Fig. 9 shows the convergence performance of each canceller when the number of training symbols changes from 5 to 100 . When a canceller is trained sufficiently, it achieves a cancellation performance close to $50 \mathrm{~dB}$ because the INR is $50 \mathrm{~dB}$ in these results. In these figures, we do not include the performance of the HaRx canceller because the power of the signal distorted by the LNA is lower than the thermal noise level in the situation, and the performance of the HaRx canceller is almost the same as that of the Hammerstein canceller. To achieve $45 \mathrm{~dB}$ cancellation, the Hammerstein canceller needs 50 OFDM symbols, while the proposed canceller requires only five or fewer. From Fig. 10, we can confirm that the proposed canceller achieves around $47 \mathrm{~dB}$ cancellation with three training OFDM symbols and has a convergence speed more than 16 times higher than the Hammerstein canceller. Although the models of the two cancellers are different, the number of parameters is a rough measure of the convergence speed of both. The Hammerstein 


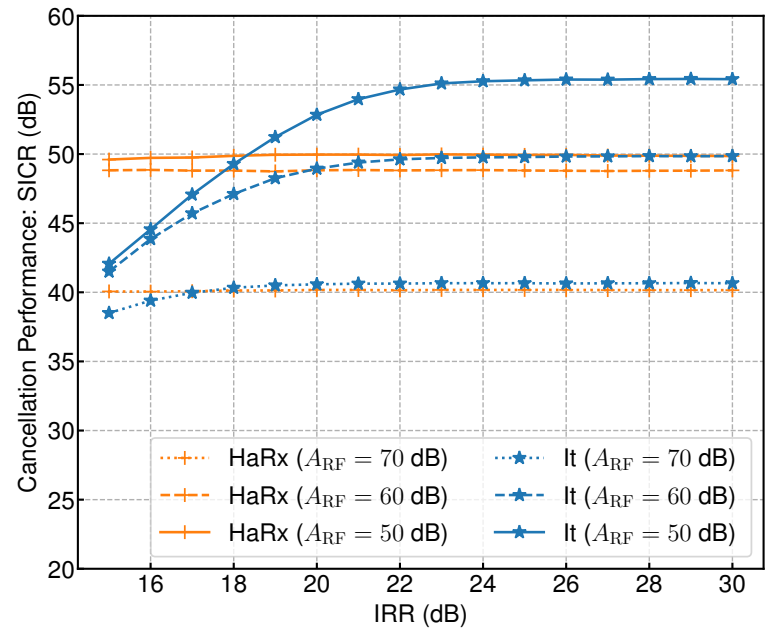

Fig. 11. Performance of each canceller with 100 training symbols and different IRR values. In this result, $M_{\mathrm{n}}=1, L=3$, and $K=8$.

canceller has $20 \times 48=960$ coefficients. In contrast, the proposed canceller has $48+1+3+1+3=56$ coefficients, which are approximately $1 / 17$ less than those of the Hammerstein canceller. From the comparison of the number of parameters, it can be shown that the proposed canceller has a faster convergence speed than the Hammerstein one.

\section{Limitations of the Proposed Canceller}

It is important to evaluate under what condition the proposed canceller performs worse than the Hammerstein canceller. Fig. 7 and Fig. 8 show comparisons of the performance of the cancellers when the transmission power and the gain of the self-interference channel $A_{\mathrm{RF}}$ are changed, respectively. We confirm that the proposed canceller achieves the same or better performance than the Hammerstein canceller even if the nonlinearities of the PA and LNA are severe. We further discuss the limitation of the proposed canceller considering the performance of cancellers at various IRR values, as shown in Fig. 11. When the IQ imbalance is so strong that the IRR is less than $20 \mathrm{~dB}$, the performance of the proposed canceller deteriorates significantly. When the IRR is very low, the assumption $\left|b_{\mathrm{rx}} b_{\mathrm{tx}}^{*}\right| \ll 1$, which is necessary to obtain (38) and derive the imbalance estimation stage, no longer holds. Thus, the proposed canceller cannot estimate the coefficients of the IQ imbalance well with severe IQ imbalance. The IRR of less than $20 \mathrm{~dB}$ is extremely severe case [55], [56], so that the degradation will hardly be a problem on actual terminals.

\section{Computational Cost}

The computational cost is an important performance indicator of self-interference cancellers. The parameters, and their values, related to computational cost are listed in TABLE VIII. The number of both multiplications and additions for training the Hammerstein canceller with 20 basis functions can be expressed as $20 M N_{\mathrm{tr}} N_{\mathrm{sym}} \approx 3.07 \times 10^{7}$ when we perform the pseudo-inverse matrix of the LS algorithm a priori [23].
TABLE VIII

PARAMETERS, AND THEIR VALUES, FOR COMPARING COMPUTATIONAL Costs

\begin{tabular}{c|c|c}
\hline \hline Parameter & Proposed & Hammerstein \\
\hline$N_{\text {sym }}$ & \multicolumn{2}{|c}{$(64+16) \times 8=640$} \\
$N_{\mathrm{fft}}$ & \multicolumn{2}{|c}{$64 \times 8=512$} \\
$N_{\mathrm{sc}}$ & \multicolumn{2}{|c}{52} \\
$M$ & \multicolumn{2}{|c}{48} \\
$P$ & 3 & 7 \\
\hline$N_{\mathrm{tr}}$ & 1 & 50 \\
$M_{\mathrm{n}}$ & 8 & - \\
$K$ & 3 & - \\
$L$ & 3
\end{tabular}

If the Hammerstein canceller is trained with a data signal instead of a training signal, substantial computational power is required due to the pseudo-inverse matrix. For training with the data signal, the least mean squares (LMS) algorithm is a practical solution. The number of both multiplications and additions of the LMS algorithm for the Hammerstein canceller with 20 basis functions is $40 M$ per sample [28]. For training with 50 OFDM symbols, the LMS algorithm needs $6.14 \times 10^{7}$ multiplications and additions, which are more than those required by the LS algorithm with the pre-calculation technique. Moreover, the LMS algorithm is less convergent than the LS algorithm, so it needs a longer signal compared to the LS algorithm; more specifically, it requires more than 50 OFDM symbols and $6.14 \times 10^{7}$ multiplications and additions. The recursive-LS algorithm exhibits fast convergence like the LS algorithm, but its computational cost is not practical for the time-domain Hammerstein canceller [28].

We have discussed the computational cost of the proposed scheme in Section III-F. According to the values in TABLE VIII, the number of MULs/DIVs, ADDs/SUBs, and $\sqrt{x}$ required for training the proposed canceller are approximately $1.23 \times 10^{6}, 1.28 \times 10^{6}$, and $3.49 \times 10^{4}$, respectively. Note that this cost is the same whether the used signal is a training signal or a data signal because we cannot implement any precalculation techniques for the proposed canceller. There is a multiplier-free algorithm to compute $\sqrt{x}$ [61], which needs only 10 times addition for 14 bit fixed-point real value, which requires accuracy of $10^{-5}$ or less. Thus, the total cost to compute $3.49 \times 10^{4}$ times $\sqrt{x}$ is sufficiently smaller than the total cost of $1.28 \times 10^{6}$ ADDs/SUBs. Therefore, the proposed canceller can complete training with less than one-tenth of the computational cost required to train the Hammerstein canceller.

For self-interference cancellation of an OFDM symbol, the proposed canceller needs about $4.73 \times 10^{4}$ MULs/DIVs, $4.10 \times 10^{4} \mathrm{ADDs} / \mathrm{SUBs}$, and $1.92 \times 10^{3}$ square-roots. The computational cost of the Hammerstein canceller for cancellation of an OFDM symbol is $20 M N_{\mathrm{sym}} \approx 6.14 \times 10^{5}$ multiplications and additions. Thus, the proposed canceller can significantly reduce the computational cost of cancellation compared with the Hammerstein canceller.

\section{CONClusion}

We have introduced operators that express characteristics of each RF component in a minimum form and have de- 
rived a nonlinear self-interference signal model. Then, we have proposed a novel nonlinear self-interference canceller to effectively reduce the nonlinear self-interference caused by TX and RX IQ mixers, the PA, and the LNA. The estimation process of the proposed canceller consists of three stages, which estimate the characteristic of the corresponding operator of each RF component. Simulation results show that the proposed canceller can estimate and remove the received selfinterference signal, which is distorted by the LNA. In addition, the proposed canceller achieves higher cancellation performance with fewer learning symbols and lower computational cost than the Hammerstein canceller.

\section{APPENDIX}

\section{A. Derivations of (26)-(30)}

The relation of the input $x$ and output $y$ of an estimated nonlinear amplifier $\diamond \in\{\mathrm{tx}, \mathrm{rx}\}$ can be written as

$$
\begin{aligned}
y & =\hat{\mathcal{D}}_{\diamond, l} x=\sum_{p=1,3, \cdots}^{P} \hat{d}_{\diamond, p, l} x|x|^{p-1} \\
& =\hat{f}_{\diamond, l}(|x|) \frac{x}{|x|} \exp \left(j \hat{\phi}_{\diamond, l}(|x|)\right),
\end{aligned}
$$

where $\hat{f}_{\diamond, l}(|x|)$ and $\hat{\phi}_{\diamond, l}(|x|)$ are the amplitude-to-amplitude (AM/AM) modulation and amplitude-to-phase (AM/PM) modulation of the estimated amplifier, respectively. The amplitude of the output $y$ can be written as

$$
|y|=\hat{f}_{\diamond, l}(|x|)=\left.\left|\sum_{p=1,3, \cdots}^{P} \hat{d}_{\diamond, p, l} x\right| x\right|^{p-1} \mid .
$$

Then, we can compute the amplitude of the input signal $|x|$ from the amplitude of the output signal $|y|$ by Newton's method as

$$
r_{k+1}=r_{k}-\frac{\left|u_{k}\right|-|y|}{\hat{f}_{\diamond, l}^{\prime}\left(r_{k}\right)}
$$

where

$$
\begin{aligned}
\hat{f}_{\diamond, l}^{\prime}(r) & =\frac{\operatorname{Re}\left[u_{k}\right] \operatorname{Re}\left[v_{k}\right]+\operatorname{Im}\left[u_{k}\right] \operatorname{Im}\left[v_{k}\right]}{\left|u_{k}\right|} \\
u_{k} & =\hat{\mathcal{D}}_{\diamond, l} r_{k}, \\
v_{k} & =\left.\frac{d}{d r}\left(\hat{\mathcal{D}}_{\diamond, l} r\right)\right|_{r=r_{k}}=\sum_{p=1,3, \cdots}^{P} \hat{d}_{\diamond, p, l} p r_{k}^{p-1} .
\end{aligned}
$$

Then, (28) is derived from (68)-(71). Moreover, from (66), the phase of input signal can be written as

$$
\frac{x}{|x|}=\frac{y}{\hat{f}_{\diamond, l}(|x|)} \exp \left(-j \phi_{\diamond, l}(|x|)\right) \approx \frac{y}{|y|} \frac{\left|u_{M_{\mathrm{n}}}\right|}{u_{M_{\mathrm{n}}}} .
$$

The final approximation of (72) holds when $|x| \approx r_{M_{\mathrm{n}}}$. Finally, (26) is derived from (28) and (72).

\section{REFERENCES}

[1] J. I. Choi, M. Jain, K. Srinivasan, P. Levis, and S. Katti, "Achieving single channel, full duplex wireless communication," in Proc. ACM MobiCom'10, 2010, pp. 1-12.

[2] A. Aijaz and P. Kulkarni, "Protocol design for enabling full-duplex operation in next-generation IEEE 802.11 WLANs," IEEE Syst. J., vol. 12, no. 4, pp. 3438-3449, Dec. 2018.

[3] M. Kaneko, "Throughput analysis of CSMA with imperfect collision detection in full duplex-enabled WLAN," IEEE Wireless Commun. Lett., vol. 6, no. 4, pp. 490-493, Aug. 2017.

[4] G. Liu, F. R. Yu, H. Ji, V. C. M. Leung, and X. Li, "In-band full-duplex relaying: A survey, research issues and challenges," IEEE Commun. Surveys Tuts., vol. 17, no. 2, pp. 500-524, 2015.

[5] B. Ma, H. Shah-Mansouri, and V. W. S. Wong, "Full-duplex relaying for D2D communication in millimeter wave-based 5G networks," IEEE Trans. Wireless Commun., vol. 17, no. 7, pp. 4417-4431, Jul. 2018.

[6] E. Hossain and M. Hasan, "5G cellular: key enabling technologies and research challenges," IEEE Instrum. Meas. Mag., vol. 18, no. 3, pp. 11-21, Jun. 2015

[7] Z. Zhang, X. Chai, K. Long, A. V. Vasilakos, and L. Hanzo, "Full duplex techniques for $5 \mathrm{G}$ networks: self-interference cancellation, protocol design, and relay selection," IEEE Commun. Mag., vol. 53, no. 5, pp. 128-137, May 2015.

[8] R.-A. Pitaval, O. Tirkkonen, R. Wichman, K. Pajukoski, E. Lahetkangas, and E. Tiirola, "Full-duplex self-backhauling for small-cell 5G networks," IEEE Wireless Commu., vol. 22, no. 5, pp. 83-89, Oct. 2015.

[9] D. Kim, H. Lee, and D. Hong, "A survey of in-band full-duplex transmission: From the perspective of PHY and MAC layers," IEEE Commun. Surveys Tuts., vol. 17, no. 4, pp. 2017-2046, 2015.

[10] K. E. Kolodziej, B. T. Perry, and J. S. Herd, "In-band full-duplex technology: Techniques and systems survey," IEEE Trans. Microw. Theory Tech., pp. 1-17, 2019.

[11] E. Everett, A. Sahai, and A. Sabharwal, "Passive self-interference suppression for full-duplex infrastructure nodes," IEEE Trans. Wireless Commun., vol. 13, no. 2, pp. 680-694, Feb. 2014.

[12] D. Bharadia, E. McMilin, and S. Katti, "Full duplex radios," in Proc. ACM SIGCOMM'13, vol. 43, no. 4, 2013, pp. 375-386.

[13] M. Jain, J. I. Choi, T. Kim, D. Bharadia, S. Seth, K. Srinivasan, P. Levis, S. Katti, and P. Sinha, "Practical, real-time, full duplex wireless," in Proc. ACM MobiCom'11, 2011, p. 301.

[14] J. Tamminen, M. Turunen, D. Korpi, T. Huusari, Y.-S. Choi, S. Talwar, and M. Valkama, "Digitally-controlled RF self-interference canceller for full-duplex radios," in Proc. 24th European Signal Process. Conf. (EUSIPCO), vol. 2016-Novem, Aug. 2016, pp. 783-787.

[15] J. Zhou, T.-H. Chuang, T. Dinc, and H. Krishnaswamy, "Integrated wideband self-interference cancellation in the RF domain for FDD and full-duplex wireless," IEEE J. Solid-State Circuits, vol. 50, no. 12, pp. 3015-3031, Dec. 2015.

[16] D. Korpi, Y.-S. Choi, T. Huusari, L. Anttila, S. Talwar, and M. Valkama, "Adaptive nonlinear digital self-interference cancellation for mobile inband full-duplex radio: Algorithms and RF measurements," in Proc. IEEE GLOBECOM, Dec. 2015, pp. 1-7.

[17] M. Duarte, C. Dick, and A. Sabharwal, "Experiment-driven characterization of full-duplex wireless systems," IEEE Trans. Wireless Commun., vol. 11, no. 12, pp. 4296-4307, Dec. 2012.

[18] J.-H. Lee, J. won Choi, J.-H. Jung, S.-C. Kim, and Y.-H. Kim, "Analog cancellation for full-duplex wireless in multipath self-interference channels," IEICE Trans. Commun., vol. E98.B, no. 4, pp. 646-652, 2015.

[19] M. Sakai, H. Lin, and K. Yamashita, "Adaptive cancellation of selfinterference in full-duplex wireless with transmitter IQ imbalance," in Proc. IEEE GLOBECOM, Dec. 2014, pp. 3220-3224.

[20] — , "Self-interference cancellation in full-duplex wireless with IQ imbalance," Elsevier Phy. Commun., vol. 18, pp. 2-14, Mar. 2016.

[21] Y. Liu, X. Quan, W. Pan, and Y. Tang, "Digitally assisted analog interference cancellation for in-band full-duplex radios," IEEE Commun. Lett., vol. 21, no. 5, pp. 1079-1082, May 2017.

[22] D. Korpi, L. Anttila, V. Syrjälä, and M. Valkama, "Widely linear digital self-interference cancellation in direct-conversion full-duplex transceiver," IEEE J. Sel. Areas Commun., vol. 32, no. 9, pp. 16741687, Sep. 2014.

[23] L. Anttila, D. Korpi, V. Syrjälä, and M. Valkama, "Cancellation of power amplifier induced nonlinear self-interference in full-duplex transceivers," in Proc. 47th Asilomar Conf. Signals, Syst., Comput., Nov. 2013, pp. 16. 
[24] L. Anttila, D. Korpi, E. Antonio-Rodriguez, R. Wichman, and M. Valkama, "Modeling and efficient cancellation of nonlinear selfinterference in MIMO full-duplex transceivers," in Proc. IEEE GC Wkshps, Dec. 2014, pp. 777-783.

[25] M. Heino, D. Korpi, T. Huusari, E. Antonio-Rodriguez, S. Venkatasubramanian, T. Riihonen, L. Anttila, C. Icheln, K. Haneda, R. Wichman, and M. Valkama, "Recent advances in antenna design and interference cancellation algorithms for in-band full duplex relays," IEEE Commun. Mag., vol. 53, no. 5, pp. 91-101, May 2015.

[26] D. Korpi, T. Huusari, Y.-S. Choi, L. Anttila, S. Talwar, and M. Valkama, "Digital self-interference cancellation under nonideal RF components: Advanced algorithms and measured performance," in Proc. IEEE SPAWC, Jun. 2015, pp. 286-290.

[27] K. Komatsu, Y. Miyaji, and H. Uehara, "Frequency-domain Hammerstein self-interference canceller for in-band full-duplex OFDM systems," in Proc. IEEE WCNC, Mar. 2017.

[28] — "Basis function selection of frequency-domain Hammerstein selfinterference canceller for in-band full-duplex wireless communications," IEEE Trans. Wireless Commun., vol. 17, no. 6, pp. 3768-3780, Jun. 2018.

[29] L. Tian, S. Wang, Z. Cheng, and X. Bu, "All-digital self-interference cancellation in zero-IF full-duplex transceivers," China Communications, vol. 13, no. 11, pp. 27-34, Nov. 2016.

[30] P. P. Campo, D. Korpi, L. Anttila, and M. Valkama, "Nonlinear digital cancellation in full-duplex devices using spline-based hammerstein model," in Proc. IEEE GC Wkshps, Dec. 2018, pp. 1-7.

[31] S. Li and R. D. Murch, "An investigation into baseband techniques for single-channel full-duplex wireless communication systems," IEEE Trans. Wireless Commun., vol. 13, no. 9, pp. 4794-4806, Sep. 2014.

[32] E. Ahmed and A. M. Eltawil, "All-digital self-interference cancellation technique for full-duplex systems," IEEE Trans. Wireless Commun., vol. 14, no. 7, pp. 3519-3532, Jul. 2015.

[33] J. Li, H. Zhang, and M. Fan, "Digital self-interference cancellation based on independent component analysis for co-time co-frequency full-duplex communication systems," IEEE Access, vol. 5, pp. 10222-10231, 2017.

[34] E. Ahmed, A. M. Eltawil, and A. Sabharwal, "Self-interference cancellation with nonlinear distortion suppression for full-duplex systems," in Proc. 47th Asilomar Conf. Signals, Syst., Comput., Nov. 2013, pp. 1199-1203.

[35] — , "Self-interference cancellation with phase noise induced ICI suppression for full-duplex systems," in Proc. IEEE GLOBECOM, Dec. 2013, pp. 3384-3388.

[36] R. Li, A. Masmoudi, and T. Le-Ngoc, "Self-interference cancellation with nonlinearity and phase-noise suppression in full-duplex systems," IEEE Trans. Veh. Technol., vol. 67, no. 3, pp. 2118-2129, Mar. 2018.

[37] M. A. Islam and B. Smida, "A comprehensive self-interference model for single-antenna full-duplex communication systems," in Proc. IEEE ICC, May 2019, pp. 1-7.

[38] D. Korpi, M. Turunen, L. Anttila, and M. Valkama, "Modeling and cancellation of self-interference in full-duplex radio transceivers: Volterra series-based approach," in Proc. IEEE ICC Wkshps, May 2018, pp. 1-6.

[39] J. Liu, H. Quan, Z. Li, H. Sun, and D. Yuan, "Digital nonlinear selfinterference cancellation based on LMS-Volterra algorithm," in Proc. 3rd Int. Conf. Inf. Sci. Control Eng. (ICISCE), Jul. 2016, pp. 1298-1302.

[40] T. Riihonen, S. Werner, and R. Wichman, "Mitigation of loopback selfinterference in full-duplex MIMO relays," IEEE Trans. Signal Process., vol. 59, no. 12, pp. 5983-5993, Dec. 2011.

[41] H. Q. Ngo, H. A. Suraweera, M. Matthaiou, and E. G. Larsson, "Multipair full-duplex relaying with massive arrays and linear processing," IEEE J. Sel. Areas Commun., vol. 32, no. 9, pp. 1721-1737, Sep. 2014.

[42] D. Liu, B. Zhao, F. Wu, S. Shao, X. Pu, and Y. Tang, "Semi-blind SI cancellation for in-band full-duplex wireless communications," IEEE Commun. Lett., vol. 22, no. 5, pp. 1078-1081, May 2018.

[43] X. Quan, Y. Liu, D. Chen, S. Shao, Y. Tang, and K. Kang, "Blind nonlinear self-interference cancellation for wireless full-duplex transceivers," IEEE Access, vol. 6, pp. 37725-37737, 2018.

[44] M. Grimm, M. Allen, J. Marttila, M. Valkama, and R. Thoma, "Joint mitigation of nonlinear RF and baseband distortions in wideband directconversion receivers," IEEE Trans. Microw. Theory Tech., vol. 62, no. 1 , pp. 166-182, Jan. 2014.

[45] M. Valkama, A. S. hagh ghadam, L. Anttila, and M. Renfors, "Advanced digital signal processing techniques for compensation of nonlinear distortion in wideband multicarrier radio receivers," IEEE Trans. Microw. Theory Tech., vol. 54, no. 6, pp. 2356-2366, Jun. 2006.

[46] E. A. Keehr and A. Hajimiri, "Equalization of third-order intermodulation products in wideband direct conversion receivers," IEEE J. SolidState Circuits, vol. 43, no. 12, pp. 2853-2867, Dec. 2008.
[47] Q. Zou, M. Mikhemar, and A. H. Sayed, "Digital compensation of crossmodulation distortion in software-defined radios," IEEE J. Sel. Topics Signal Process., vol. 3, no. 3, pp. 348-361, Jun. 2009.

[48] T. Riihonen, A. Balakrishnan, K. Haneda, S. Wyne, S. Werner, and R. Wichman, "Optimal eigenbeamforming for suppressing selfinterference in full-duplex MIMO relays," in Proc. 45th Аnnu. CISS, Mar. 2011.

[49] K. Satyanarayana, M. El-Hajjar, P.-H. Kuo, A. Mourad, and L. Hanzo, "Hybrid beamforming design for full-duplex millimeter wave communication," IEEE Trans. Veh. Technol., vol. 68, no. 2, pp. 1394-1404, Feb. 2019.

[50] K. Satyanarayana, M. El-Hajjar, A. Mourad, and L. Hanzo, "Multi-user full duplex transceiver design for mmWave systems using learning-aided channel prediction," IEEE Access, vol. 7, pp. 66 068-66 083, 2019.

[51] T. Riihonen, S. Werner, and R. Wichman, "Transmit power optimization for multiantenna decode-and-forward relays with loopback selfinterference from full-duplex operation," in Proc. 45th Asilomar Conf. Signals, Syst., Comput., Nov. 2011, pp. 1408-1412.

[52] — , "Hybrid full-duplex/half-duplex relaying with transmit power adaptation," IEEE Trans. Wireless Commun., vol. 10, no. 9, pp. 30743085, Sep. 2011.

[53] M. Valkama and M. Renfors, "Digital filter design for I/Q imbalance compensation," in Proc. 10th European Signal Process. Conf. (EUSIPCO), Sep. 2000, pp. 1-4.

[54] S. Benedetto and E. Biglieri, Principles of Digital Transmission: With Wireless Applications. Norwell, MA, USA: Kluwer Academic Publishers, 1999.

[55] M. Valkama, M. Renfors, and V. Koivunen, "Advanced methods for I/Q imbalance compensation in communication receivers," IEEE Trans. Signal Process., vol. 49, no. 10, pp. 2335-2344, 2001.

[56] A. Tarighat and A. Sayed, "Joint compensation of transmitter and receiver impairments in OFDM systems," IEEE Trans. Wireless Commun., vol. 6, no. 1, pp. 240-247, Jan. 2007.

[57] T. Bäckstöm, "Vandermonde factorization of Toeplitz matrices and applications in filtering and warping," IEEE Trans. Signal Process., vol. 61 , no. 24, pp. 6257-6263, Dec. 2013.

[58] G. Heinig and K. Rost, "Fast algorithms for Toeplitz and Hankel matrices," Linear Algebra and its Applications, vol. 435, no. 1, pp. 1-59, 2011.

[59] R. Hunger, "Floating point operations in matrix-vector calculus," Technische Universität München, Associate Institute for Signal Processing, Tech. Rep., 2007.

[60] C. Rapp, "Effects of HPA-nonlinearity on a 4-DPSK/OFDM-signal for a digital sound broadcasting system," in Proc. the Second Europian Conf. on Satellite Commun., Oct. 1991, pp. 179-184.

[61] F. Auger, Z. Lou, B. Feuvrie, and F. Li, "Multiplier-free divide, square root, and log algorithms [DSP tips and tricks]," IEEE Signal Process. Mag., vol. 28, no. 4, pp. 122-126, Jul. 2011.

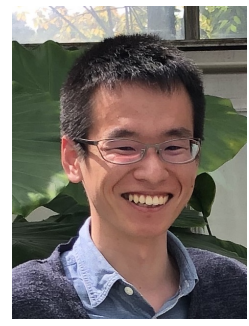

Kazuki Komatsu (S'17) was born in Nara, Japan, in 1994. He received B.E. and M.E. degrees from Toyohashi University of Technology, Japan, in 2016 and 2018, respectively. He is currently a doctoral student in the Graduate School of Engineering, Toyohashi University of Technology. Since 2019, he has been a Research Fellowship for Young Scientists of the Japan Society for the Promotion of Science (JSPSDC2). His current research interests include in-band full-duplex communications. He is a member of the IEICE. He received the IEEE Nagoya Section Excellent Student Award in 2016 and the IEEE Communications Society Young Professionals Best Paper Award in 2017. 


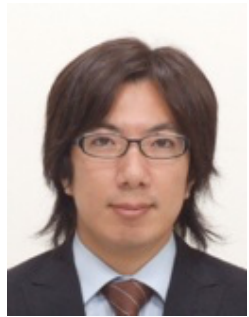

Yuichi Miyaji (S'10-M'13) was born in Kagoshima, Japan, in 1984. He received B.E., M.E., and Ph.D degrees from Toyohashi University of Technology, Japan, in 2007, 2009, and 2013, respectively. Since 2013, he has been an Assistant Professor at the Department of Electrical and Electronic Information Engineering, Toyohashi University of Technology. His current research interests include wireless networks and in-band full-duplex communications. He is a member of the IEICE and IPSJ. He received the IEICE Young Researcher's Award in 2016 and the IEICE Communications Society: Distinguished Contributions Award in 2016 and 2019 .

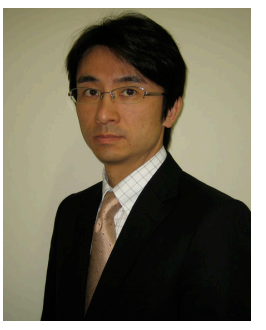

Hideyuki Uehara (S'92-M'97) received B.E., M.E., and $\mathrm{Ph} . \mathrm{D}$. degrees in electrical engineering from Keio University, Yokohama, Japan, in 1992, 1994, and 1997, respectively. Since 1997, he has been with Toyohashi University of Technology, Japan, where he is currently a Professor at the Department of Electrical and Electronic Information Engineering. From 2002 to 2003, he was a Visiting Researcher with the Advanced Telecommunications Research Institute International (ATR), Kyoto, Japan. His current research interests include wireless access and multihop communications. He is a senior member of the IEICE. He received the Distinguished Contributions Award from the IEICE Communications Society in 2002, 2006, 2011, 2012, and 2016. He served as an Editor of the Editorial Committee for the IEICE Transactions on Communications from 2010 to 2011 and a Vice-Chair of the Editorial Board for the IEICE Communications Society from 2014 to 2015 . From 2017 to 2018, he served as a Treasurer of the IEEE Nagoya Section. 